\title{
Evolving neoantigen profiles in colorectal cancers with DNA repair defects
}

\author{
Giuseppe Rospo ${ }^{1 \dagger}$, Annalisa Lorenzato ${ }^{1,2+}$, Nabil Amirouchene-Angelozzi ${ }^{3}$, Alessandro Magri ${ }^{1,2}$, \\ Carlotta Cancelliere ${ }^{1}$, Giorgio Corti ${ }^{1}$, Carola Negrino ${ }^{1,2}$, Vito Amodio ${ }^{1,2}$, Monica Montone ${ }^{1}$, Alice Bartolini ${ }^{1}$, \\ Ludovic Barault 1,2, Luca Novara', Claudio Isella', Enzo Medico ${ }^{1,2}$, Andrea Bertotti ${ }^{1,2}$, Livio Trusolino ${ }^{1,2}$, \\ Giovanni Germano ${ }^{1,2}$, Federica Di Nicolantonio ${ }^{1,2}$ and Alberto Bardelli ${ }^{12^{*}}$ (i)
}

\begin{abstract}
Background: Neoantigens that arise as a consequence of tumor-specific mutations can be recognized by $T$ lymphocytes leading to effective immune surveillance. In colorectal cancer (CRC) and other tumor types, a high number of neoantigens is associated with patient response to immune therapies. The molecular processes governing the generation of neoantigens and their turnover in cancer cells are poorly understood. We exploited CRC as a model system to understand how alterations in DNA repair pathways modulate neoantigen profiles over time.

Methods: We performed whole exome sequencing (WES) and RNA sequencing (RNAseq) in CRC cell lines, in vitro and in vivo, and in CRC patient-derived xenografts (PDXs) to track longitudinally genomic profiles, clonal evolution, mutational signatures, and predicted neoantigens.

Results: The majority of CRC models showed remarkably stable mutational and neoantigen profiles; however, those carrying defects in DNA repair genes continuously diversified. Rapidly evolving and evolutionary stable CRCs displayed characteristic genomic signatures and transcriptional profiles. Downregulation of molecules implicated in antigen presentation occurred selectively in highly mutated and rapidly evolving CRC.

Conclusions: These results indicate that CRCs carrying alterations in DNA repair pathways display dynamic neoantigen patterns that fluctuate over time. We define CRC subsets characterized by slow and fast evolvability and link this phenotype to downregulation of antigen-presenting cellular mechanisms. Longitudinal monitoring of the neoantigen landscape could be relevant in the context of precision medicine.
\end{abstract}

Keywords: Colorectal cancer, Neoantigen, DNA repair, Immune response, Mutational signature

\section{Background}

Anticancer therapies based on immune-checkpoint blockade are often remarkably effective but benefit only a minor fraction of cancer patients [1]. Several biomarkers of response and resistance to immune modulators have been proposed [2, 3]. Among these, the overall mutational burden (number of somatic variants per megabase $(\mathrm{Mb})$ ) and the number of predicted neoantigens were highlighted in multiple studies [4-6]. The predictive values of mutational and antigen burdens are still being evaluated in

\footnotetext{
* Correspondence: alberto.bardelli@unito.it

${ }^{\dagger}$ Giuseppe Rospo and Annalisa Lorenzato contributed equally to this work.

${ }^{1}$ Candiolo Cancer Institute, FPO-IRCCS, 10060 Candiolo (TO), Italy

${ }^{2}$ Department of Oncology, University of Turin, 10060 Candiolo (TO), Italy

Full list of author information is available at the end of the article
}

clinical settings. Both parameters are presently assessed on DNA extracted from individual tissue samples and are typically measured only once in the clinical history of each patient. Alterations in DNA repair pathways, including mutations or promoter hypermethylation of mismatch repair (MMR) effectors ( $M L H 1, M S H 2$, etc.) or DNA polymerases (polymerase $\varepsilon$ and $\delta$ ) [7], are known to increase the mutational burden and the neoantigen profiles of cancers [8]. Whether, and to what extent, neoantigen profiles evolve over time as a result of the inherent genomic instability of individual tumors is largely unknown. We recently reported that in mouse models, inactivation of DNA mismatch repair increases the mutational burden and leads to dynamic mutational profiles resulting in effective cancer immune response [9]. Here

(c) The Author(s). 2019 Open Access This article is distributed under the terms of the Creative Commons Attribution 4.0 International License (http://creativecommons.org/licenses/by/4.0/), which permits unrestricted use, distribution, and 
we exploit CRCs as a model system to understand whether mutational burden and neoantigen profile of human tumors evolve over time as a result of their distinctive genomic landscapes.

\section{Methods}

\section{CRC cell lines}

The source of each cell line is reported in Table 1. All cell lines were maintained in their original culturing conditions according to supplier guidelines. Cells were ordinarily supplemented with FBS 10\%, 2 mM L-glutamine, and antibiotics $(100 \mathrm{U} / \mathrm{mL}$ penicillin and $100 \mathrm{mg} / \mathrm{mL}$ streptomycin) and grown in a $37^{\circ} \mathrm{C}$ and $5 \% \mathrm{CO}_{2}$ air incubator. To study the evolution of cell populations, cell lines were not cloned prior to the experiment or at any subsequent time point. Cell lines were thawed in a $10-\mathrm{cm}$ dish. After thaw recovery, each cell line was screened for the absence of mycoplasma contamination and checked for its identity, referred below as quality control (QC). To preserve heterogeneity, upon thawing, individual lines were expanded to at least $10^{8}$ cells. At this point for each model, cells were counted, and the percentage of alive/dead cells was calculated. At the beginning of the experiment (T0), $4 \times 10^{7}$ live cells were distributed as follows: (A) $2 \times 10^{6}$ cells were re-plated in a $10-\mathrm{cm}$ dish for in vitro propagation, (B) $3 \times 10^{7}$ cells were used for in vivo experiments, (C) $2 \times 10^{6}$ cells were frozen, and (D) 3 pellets $\left(2 \times 10^{6}\right.$ cells each) were frozen for DNA, RNA, and protein extraction. Cells plated as in (A) were kept in culture changing medium twice a week and dividing them at a constant splitting rate, determined before initiating the experiment. In details, splitting was performed before full confluency was achieved. The number of cells that were split and the number of passages and days of culture were recorded for each cell model to calculate the doubling time. During in vitro culture, cell populations were collected at the following pre-determined time points: 30 days (T30), 60 days (T60) and 90 days (T90) from T0. At each time point, a fraction of the cells were put aside (note that this did not affect the rate of passaging described below) and pellets $\left(2 \times 10^{6}\right.$ each) were collected for DNA, RNA, and protein extraction. $\mathrm{QC}$ was repeated at each time point.

\section{Cell quality control (QC)}

Cells were screened for the absence of mycoplasma contamination using the Venor ${ }^{\circ} \mathrm{GeM}$ Classic kit (Minerva Biolabs). The identity of each cell line was checked before starting each experiment and after every genomic DNA extraction by PowerPlex ${ }^{\circ} 16$ HS System (Promega), through Short Tandem Repeats (STR) at 16 different loci (D5S818, D13S317, D7S820, D16S539, D21S11, vWA, TH01, TPOX, CSF1PO, D18S51, D3S1358, D8S1179, FGA, Penta D, Penta E, and amelogenin). Amplicons from multiplex PCRs were separated by capillary electrophoresis (3730 DNA Analyzer, Applied Biosystems) and analyzed using GeneMapper v 3.7 software (Life Technologies).

\section{Microsatellite instability (MSI) status}

The MSI status was assessed with the MSI Analysis System kit (Promega). The analysis requires a multiplex amplification of seven markers including five mononucleotide repeat markers (BAT-25, BAT-26, NR-21, NR-24, and MONO-27) and two pentanucleotide repeat markers (Penta $\mathrm{C}$ and Penta $\mathrm{D}$ ). The products were analyzed by capillary electrophoresis in a single injection (3730 DNA Analyzer, ABI capillary electrophoresis system (Applied Biosystems). Then, the results were analyzed using GeneMapper V5.0 software.

\section{DNA extraction and exome sequencing}

Genomic DNA (gDNA) was extracted from CRC cell lines, xenografts, and PDXs using Maxwell ${ }^{\circ}$ RSC Blood DNA kit (AS1400, Promega). DNA was sent to IntegraGen SA (Evry, France) that performed library preparation, exome capture, sequencing, and data demultiplexing. Final DNA libraries were pair-end sequenced on Illumina HiSeq4000 as pairedend $100 \mathrm{bp}$ reads.

\section{Mutational analysis in cell lines}

When cell lines were passaged in mice or when analyzing patient-derived xenografts, Fastq files were first processed with Xenome [10] to remove reads of mouse origin. Reads files were aligned to the human reference hg38 using BWA-mem algorithm [11], and then the "rmdup" samtools command was used to remove PCR duplicates [12]. On the resulting aligned files, we observed a median depth of $138 x$ with $98 \%$ of the targeted region covered by at least one read. Bioinformatic modules previously developed $[9,13]$ by our laboratory were used to identify single nucleotide variants (SNVs) and indels. The mutational characterization of the 64 cell lines at time point 0 was assessed by calling the alterations against the hg38 reference annotation. Then, a series of filters were used to remove germline variants and artifacts: alleles supported by only reads with the same strand, excluding start and end read positions from the count, were discarded; variants called with allelic frequency lower than $10 \%$ as well a $p$ value greater than 0.05 (binomial test calculated on allele count and depth of each sample) were excluded; common dbSNP version 147 and a panel of normal (40 samples) from previous sequencing were used to annotate and filter germline variants and sequencing artifacts. The variant calls of 45 cell lines at time point 90 and the 18 cell lines explanted from mice were performed using the allele comparison strategy between the same cell line at time 0 and time 
Table 1 Molecular, functional characteristics and source of origin of the indicated cell lines

\begin{tabular}{|c|c|c|c|c|c|c|c|}
\hline Sample & $\begin{array}{l}\text { Microsatellite } \\
\text { status }\end{array}$ & Altered MSI markers & $\begin{array}{l}\text { Genome } \\
\text { evolvability }\end{array}$ & $\begin{array}{l}\text { Split } \\
\text { ratio }\end{array}$ & $\begin{array}{l}\text { In vitro } \\
\text { doubling time }\end{array}$ & Growth rate & Source \\
\hline $\mathrm{C} 10$ & Stable & - & Stable & 0.34 & 2.33 & 0.30 & ECACC \\
\hline C106 & Stable & - & Stable & 0.35 & 2.50 & 0.28 & ECACC \\
\hline C125PM & Stable & - & Stable & 0.34 & 2.37 & 0.29 & ECACC \\
\hline C32 & Stable & - & Stable & 0.18 & 1.54 & 0.45 & ECACC \\
\hline C70 & Stable & - & Stable & 0.4 & 2.76 & 0.25 & ECACC \\
\hline C75 & Stable & - & Stable & 0.36 & 2.46 & 0.28 & ECACC \\
\hline C99 & Stable & - & NA & NA & NA & NA & ECACC \\
\hline $\mathrm{CACO} 2$ & Stable & - & Stable & 0.26 & 1.83 & 0.38 & ATCC \\
\hline CAR1 & Stable & - & Stable & 0.36 & 2.47 & 0.28 & JCRB \\
\hline CCK81 & Unstable & bat26-nr21-bat25-mono27-nr24 & Evolving & 0.34 & 2.53 & 0.27 & RIKEN \\
\hline CL14 & Stable & - & NA & NA & NA & NA & DSMZ \\
\hline COCM1 & Stable & - & Evolving & 0.3 & 2.34 & 0.30 & JCRB \\
\hline COGA1 & Unstable & bat26-mono27-nr24 & NA & NA & NA & NA & Dr. Huber ${ }^{a}$ \\
\hline COGA2 & Stable & - & Stable & 0.32 & 2.22 & 0.31 & Dr. Huber ${ }^{\mathrm{a}}$ \\
\hline COGA5 & Stable & - & Stable & 0.20 & 1.69 & 0.41 & Dr. Huber ${ }^{a}$ \\
\hline COGA8 & Stable & - & Stable & 0.22 & 1.67 & 0.41 & Dr. Huber ${ }^{a}$ \\
\hline COLO201 & Stable & - & NA & NA & NA & NA & ATCC \\
\hline COLO94H & Stable & - & Stable & 0.45 & 2.81 & 0.25 & CLS \\
\hline DIFI & Stable & - & NA & NA & NA & NA & Dr. Baselga ${ }^{b}$ \\
\hline DLD1 & Unstable & bat26-nr21-bat25-mono27-nr24 & Evolving & 0.07 & 0.98 & 0.71 & $\mathrm{NCl} 60$ \\
\hline HCA24 & Stable & - & Evolving & 0.33 & 2.22 & 0.31 & ECACC \\
\hline HCA46 & Stable & - & Stable & 0.4 & 2.41 & 0.29 & ECACC \\
\hline HCC2998 & Stable & - & Stable & 0.34 & 2.33 & 0.30 & NCl60 \\
\hline HDC114 & Stable & - & Evolving & 0.23 & 1.69 & 0.41 & $\mathrm{DKFZ}^{\mathrm{C}}$ \\
\hline HDC142 & Stable & - & Evolving & 0.35 & 2.42 & 0.29 & DKFZ \\
\hline HDC82 & Stable & - & NA & NA & NA & NA & $\mathrm{DKFZ}^{\mathrm{C}}$ \\
\hline HRA16 & Stable & - & NA & NA & NA & NA & ECACC \\
\hline $\mathrm{HROC} 24$ & Unstable & bat26-nr21-bat25-mono27-nr24 & Evolving & 0.16 & 1.23 & 0.56 & Dr. Linnebacher ${ }^{d}$ \\
\hline HROC32 & Stable & - & Stable & 0.62 & 6.07 & 0.11 & Dr. Linnebacher ${ }^{d}$ \\
\hline HROC334 & Stable & - & Stable & 0.45 & 2.83 & 0.24 & Dr. Linnebacher ${ }^{\mathrm{d}}$ \\
\hline HROC39 & Stable & - & Stable & 0.5 & 3.92 & 0.18 & Dr. Linnebacher ${ }^{d}$ \\
\hline HROC69 & Stable & - & Stable & 0.33 & 2.26 & 0.31 & Dr. Linnebacher ${ }^{d}$ \\
\hline HT115 & Stable & - & Evolving & 0.24 & 1.78 & 0.39 & ECACC \\
\hline HT29 & Stable & - & Stable & 0.16 & 1.38 & 0.50 & $\mathrm{NCl} 60$ \\
\hline HT55 & Stable & - & Stable & 0.33 & 2.21 & 0.31 & ECACC \\
\hline LIM1215 & Unstable & bat26-nr21-bat25-mono27-nr24 & Evolving & 0.15 & 1.24 & 0.56 & Dr. Whitehead ${ }^{e}$ \\
\hline LIM2099 & Stable & - & Stable & 0.33 & 2.26 & 0.31 & Dr. Whitehead ${ }^{e}$ \\
\hline LOVO & Unstable & nr21-bat25-mono27-nr24 & NA & NA & NA & NA & ATCC \\
\hline LS180 & Unstable & bat26-nr21-bat25-mono27-nr24 & Evolving & 0.25 & 1.90 & 0.37 & ATCC \\
\hline LS411N & Unstable & bat26-nr21-bat25-mono27-nr24 & Evolving & 0.32 & 2.29 & 0.30 & ATCC \\
\hline MDST8 & Stable & - & Stable & 0.15 & 1.31 & 0.53 & ECACC \\
\hline $\mathrm{NClH} 716$ & Stable & - & NA & NA & NA & NA & ATCC \\
\hline OUMS23 & Stable & - & Stable & 0.26 & 1.70 & 0.41 & JCRB \\
\hline
\end{tabular}


Table 1 Molecular, functional characteristics and source of origin of the indicated cell lines (Continued)

\begin{tabular}{|c|c|c|c|c|c|c|c|}
\hline Sample & $\begin{array}{l}\text { Microsatellite } \\
\text { status }\end{array}$ & Altered MSI markers & $\begin{array}{l}\text { Genome } \\
\text { evolvability }\end{array}$ & $\begin{array}{l}\text { Split } \\
\text { ratio }\end{array}$ & $\begin{array}{l}\text { In vitro } \\
\text { doubling time }\end{array}$ & Growth rate & Source \\
\hline OxCO3 & Stable & - & Stable & 0.23 & 1.76 & 0.39 & Dr. Cerundolo ${ }^{f}$ \\
\hline RW7213 & Stable & - & Stable & 0.4 & 2.60 & 0.27 & Dr. Arango ${ }^{g}$ \\
\hline SNU1040 & Unstable & bat26-nr21-bat25-mono27-nr24 & Evolving & 0.54 & 4.14 & 0.17 & KCLB \\
\hline SNU1181 & Stable & - & Stable & 0.62 & 4.65 & 0.15 & KCLB \\
\hline SNU1235 & Stable & - & Evolving & 0.35 & 2.33 & 0.30 & KCLB \\
\hline SNU1411 & Stable & - & Evolving & 0.44 & 2.76 & 0.25 & KCLB \\
\hline SNU1460 & Stable & - & NA & NA & NA & NA & KCLB \\
\hline SNU1684 & Unstable & bat26-nr21-bat25-mono27-nr24 & NA & NA & NA & NA & KCLB \\
\hline SNU175 & Unstable & bat26-nr21-bat25-mono27 & NA & NA & NA & NA & KCLB \\
\hline SNU283 & Stable & - & NA & NA & NA & NA & KCLB \\
\hline SNU479 & Stable & - & NA & NA & NA & NA & KCLB \\
\hline SNU81 & Stable & - & Evolving & 0.42 & 2.33 & 0.30 & $\mathrm{KCLB}$ \\
\hline SNU977 & Stable & - & Stable & 0.42 & 2.71 & 0.26 & KCLB \\
\hline SNUC1 & Stable & - & NA & NA & NA & NA & KCLB \\
\hline SW1417 & Stable & - & NA & NA & NA & NA & ATCC \\
\hline SW1463 & Stable & - & NA & NA & NA & NA & ATCC \\
\hline SW480 & Stable & - & Stable & 0.18 & 1.64 & 0.42 & ATCC \\
\hline SW837 & Stable & - & Stable & 0.27 & 2.07 & 0.34 & ATCC \\
\hline V411 & Stable & - & Stable & 0.22 & 1.91 & 0.36 & Dr. Markovitz ${ }^{h}$ \\
\hline V481 & Unstable & bat26-nr21-bat25-mono27-nr24 & NA & NA & NA & NA & Dr. Markovitz ${ }^{\text {h }}$ \\
\hline WIDR & Stable & - & NA & NA & NA & NA & Dr. Bernards ${ }^{i}$ \\
\hline
\end{tabular}

Non-commercial cell lines were provided by (a) Dr. L. A. Huber, Cell Biology/Biocenter, Medical University of Innsbruck, Innsbruck, Austria; (b) Dr. Baselga, Chairman \& Professor of Medicine, Vall d'Hebron Institute of Oncology (V.H.I.O.), Vall d' Hebron University Hospital, Barcelona, Spain; (c) Dr. M. Schawb, Division of Tumour Genetics - B030 German Cancer Research Center (DKFZ), Heidelberg, Germany; (d) Dr. M. Linnebacher, Division of Molecular Oncology and Immunotherapy, Department of General Surgery, University of Rostock, Rostock, Germany; (e) Dr. R.H. Whitehead, Depts of Medicine, Cell and Developmental Biology and Cancer Biology, Vanderbilt University, Nashville, USA; (f) Dr. V. Cerundolo, Nuffield Dept of Clinical Medicine, John Radcliffe Hospital, Oxford, UK; (g) Dr. D. Arango, Group of Molecular Oncology, Nanomedicine Research Program, Molecular Biology and Biochemistry Research Center, CIBBIM Nanomedicine, Vall d'Hebron, Barcelona, Spain; (h) Dr. S. Markovitz, Case Comprehensive Cancer Center, Division of Hematology-Oncology, Department of Medicine, Case Western Reserve University, Cleveland, USA; (i) Dr. R. Bernards, Division of Molecular Carcinogenesis B7, Netherlands Cancer Institute, Amsterdam, The Netherlands.

point 90 and xenograft respectively. Only variants present at time point 90 (or in xenograft) were kept. Artifact removal was employed as described above. To calculate the tumor mutational burden (number of variants/Mb), only coding variants were considered. Those variants were used to predict neoantigens using previously published methods $[9,14]$. Briefly, RNAseq data were used as input of "OptitypePipeline" [15] to assess the HLA status of each sample at time point 0 , then NetMHC 4.0 software [16] was employed to analyze mutated peptides derived from variant calls using kmer of 8-11 length. Next, for each SNV, we modified the corresponding cDNA in the selected position and we examined the $5^{\prime}$ and $3^{\prime}$ context. The latter was set taking into account the length (in terms of amino acids) with which the putative antigen could bind HLA. We translated the cDNA and feed mutant peptide to NetMHC with the proper HLA(s). For frameshifts, we applied the same approach considering every possible peptide generated by the new frame. Finally, RNAseq data were used to annotate and then filter according to expression values (fragments per kilobase million $($ FPKM) > 10). Only predicted neoantigens with a strong binding affinity (Rank < 0.5$)$ were considered for further analysis.

\section{Mutational analysis of patient-derived xenograft}

WES of patient-derived xenografts was performed at IntegraGen SA (Evry, France). Sequenced samples included a microsatellite stable (MSS), a microsatellite unstable (MSI), and a POLE mutant case $(5,7$, and 6 respectively). Samples were analyzed with the same bioinformatic pipeline applied to cell lines, and murine reads were first removed using Xenome [10]. A median depth of $130 \mathrm{x}$ and with $98 \%$ of the targeted region covered by at least one read was observed. All 18 PDX samples were characterized by calling alterations against the hg38 reference annotation. For each generation, with the exception of the first one, the mutational evolution was inferred by subtracting the mutations of the previous generation. Second-generation samples were compared to 
the first-generation samples, samples from the third generation were compared to the 2 nd generation samples, and so on.

\section{Ploidy estimation}

Gene copy-number $(\mathrm{GCN})$ was calculated in a two-step approach: initially, we treated cell lines as diploid and considered the median read depth of all coding regions as the level for $2 \mathrm{~N}$ ploidy. We also calculated the median read depth for every gene. The ratio between the two median values was then considered as the relative GCN. In the second step, to estimate the overall ploidy, we segmented all chromosomes using a custom script that implements circular binary segmentation. Finally, we exploited the distribution of allelic frequencies for individual segments to assess the absolute GCN. This was necessary since distinct ploidy levels have different expected distributions. For example, a $2 \mathrm{~N}$ ploidy status has a bell-shaped curve with a peak of $50 \%$ and a $3 \mathrm{~N}$ ploidy is expected to have two peaks on $33 \%$ and $66 \%$.

\section{Mutational signature}

Mutational signatures were calculated using the web application "Mutational Signatures in Cancer" (MuSiCa) [17]. The profile of each signature is calculated using the six substitution subtypes: $\mathrm{C}>\mathrm{A}, \mathrm{C}>\mathrm{G}, \mathrm{C}>\mathrm{T}, \mathrm{T}>\mathrm{A}, \mathrm{T}>\mathrm{C}$, and $\mathrm{T}>\mathrm{G}$ (all substitutions are referred to by the pyrimidine of the mutated Watson-Crick base pair). Information on nucleotides $5^{\prime}$ and $3^{\prime}$ to each mutated base are incorporated to generate 96 possible mutation types. For each sample, a tab-separated value file was created with chromosome, position, reference, and alternate alleles. Only samples with at least 10 mutations were included. The output file of $\mathrm{MuSiCa}$ that includes the contribution values of 30 signatures [18] was used to create a clustermap with seaborn, a Python data visualization library, setting Euclidean metric and the average linkage method.

\section{Doubling time}

Cell lines were passaged in vitro for a minimum of 85 to a maximum of 103 days. Each passage was performed before full confluency was achieved, and the total number of doublings was annotated for each cell model. Two parameters, number of passages $(n)$ and days of culture $(t)$, were used to estimate the growth rate (GR) and the doubling time (DT) assuming that every division is an independent random event; probability distribution of division is equal for all cells and it is an exponential distribution; and the number of cells in each plate before confluence is fixed $(K)$. The growth rate is defined as $\mathrm{GR}=\log _{n}(2) \div \mathrm{DT}$ [19] . The estimated number of cells at time $t$ is defined as $N(t)=N(0) \times e^{(\mathrm{GR} \times t)}$ where $N(0)$ is the number of cells at time 0 . Therefore, $\mathrm{GR}=\log _{n}(N(t) \div N(0)) \div t$ where
$N(t) \div N(0)=\left(K \times 2^{n}\right) \div\left(K \times 2^{0}\right)=2^{n}$ and so $\mathrm{GR}=$ $\log _{n}\left(2^{n}\right) \div t$. Finally, DT $=t \times \log _{n}(2) \div \log _{n}\left(2^{n}\right)$.

\section{RNA extraction and RNAseq analysis}

Total RNA was extracted from a pellet of CRC cells $(2 \times$ $10^{6}$ cells) using Maxwell ${ }^{\circ}$ RSC miRNA Tissue Kit (AS1460, Promega), according to the manufacturer's protocol. The quantification of RNA was performed by Thermo Scientific Nanodrop 1000 (Agilent) and Qubit 3.0 Fluorometer (Life Technologies). RNA integrity was evaluated with the Agilent 2100 Bioanalyzer using the Agilent RNA 6000 Nano Kit. Total RNA (800 ng) with RNA integrity number (RIN) score between 9 and 10 was used as input to the Illumina TruSeq RNA Sample Prep Kit v2-Set B (48Rxn), according to the manufacturer's protocol. The standard RNA fragmentation profile was used $\left(94^{\circ} \mathrm{C}\right.$ for $8 \mathrm{~min}$ for the TruSeq RNA Sample Prep Kit). PCR-amplified RNAseq library quality was assessed using the Agilent DNA 1000 kit on the Agilent 2100 BioAnalyzer and quantified using Qubit 3.0 Fluorometer (Life Technologies). Libraries were diluted to $10 \mathrm{nM}$ using Tris- $\mathrm{HCl}(10 \mathrm{mM} \mathrm{pH} 8.5)$ and then pooled together. Diluted pools were denatured according to the standard Illumina protocol, and $1.8 \mathrm{pM}$ were run on NextSeq500 using high output Reagent cartridge V2 for 150 cycles. A single-read 150-cycle run was performed. FastQ files produced by Illumina NextSeq500 were aligned using MapSplice2 [20] transcriptome-aware aligner using hg38 assembly as reference genome. The resulting BAM files were post-processed to translate genomic coordinates to transcriptomic ones and to filter out alignments carrying insertions or deletions (which RSEM does not support) or falling outside the transcriptome regions. The postprocessed BAM alignment was given as input to RSEM [21] for gene expression quantification using GENCODE v22 as gene annotation.

\section{Differential expression analysis}

The abundance quantification generated with RSEM provides the FPKM and the expected counts for each gene. The latter was used to perform genes differential expression analysis with DESeq2 $\mathrm{R}$ package (library Bioconductor) [22] given two distinct groups of interest, one of which considered as the reference. Genes were considered as differentially expressed if the adjusted $p$ value was less than 0.05 , and the $\log 2$ fold change was less or equal to -1 (if median FPKM value of the reference group was greater or equal to 10), or the $\log 2$ fold change was greater or equal to 1 (if median FPKM of the target group was greater or equal to 10). The analyses were performed between the following groups: MSI vs MSS (reference), hypermutated vs non-hypermutated (reference), and "EVOLVING-CRC" vs "STABLE-CRC" (reference). The hypermutated group included MSI and MSS POLEmutated cell lines (18 samples). EVOLVING-CRC group 
included all samples with at least 10 alterations acquired per day. A multi-factor configuration of the expression analysis was designed including extra variables of interest such as growth rates or the number of mutations normalized to doubling time.

\section{Pathway analysis}

Genes differentially expressed were then analyzed with g:Profiler [23], an online pathway analysis tool that takes a list of genes and assigns them to different families of biological functions. We set the query options to select significant biological processes only, and we retained (for further analysis) only the topmost families of the hierarchy (depth 1).

\section{Xenograft mouse model}

Each CRC cell line $\left(5 \times 10^{6}\right.$ cells $)$ was injected subcutaneously into both flanks of two 6-week-old female NOD (nonobese diabetic)/SCID (severe combined immunodeficient) mice (Charles River Laboratory). Tumor size was measured twice a week and calculated using the formula: $V=\left((d)^{2} \times(D)\right) \div 2(d=$ minor tumor axis; $D=$ major tumor axis). Tumors were explanted when they reached a volume of $1000 \mathrm{~mm}^{3}$. The investigators were not blinded, and measurements were acquired before the identification of the cages.

\section{Patient-derived mouse model}

Tissue from hepatic metastasectomy of CRC patients was collected at surgery and implanted in NOD-SCID

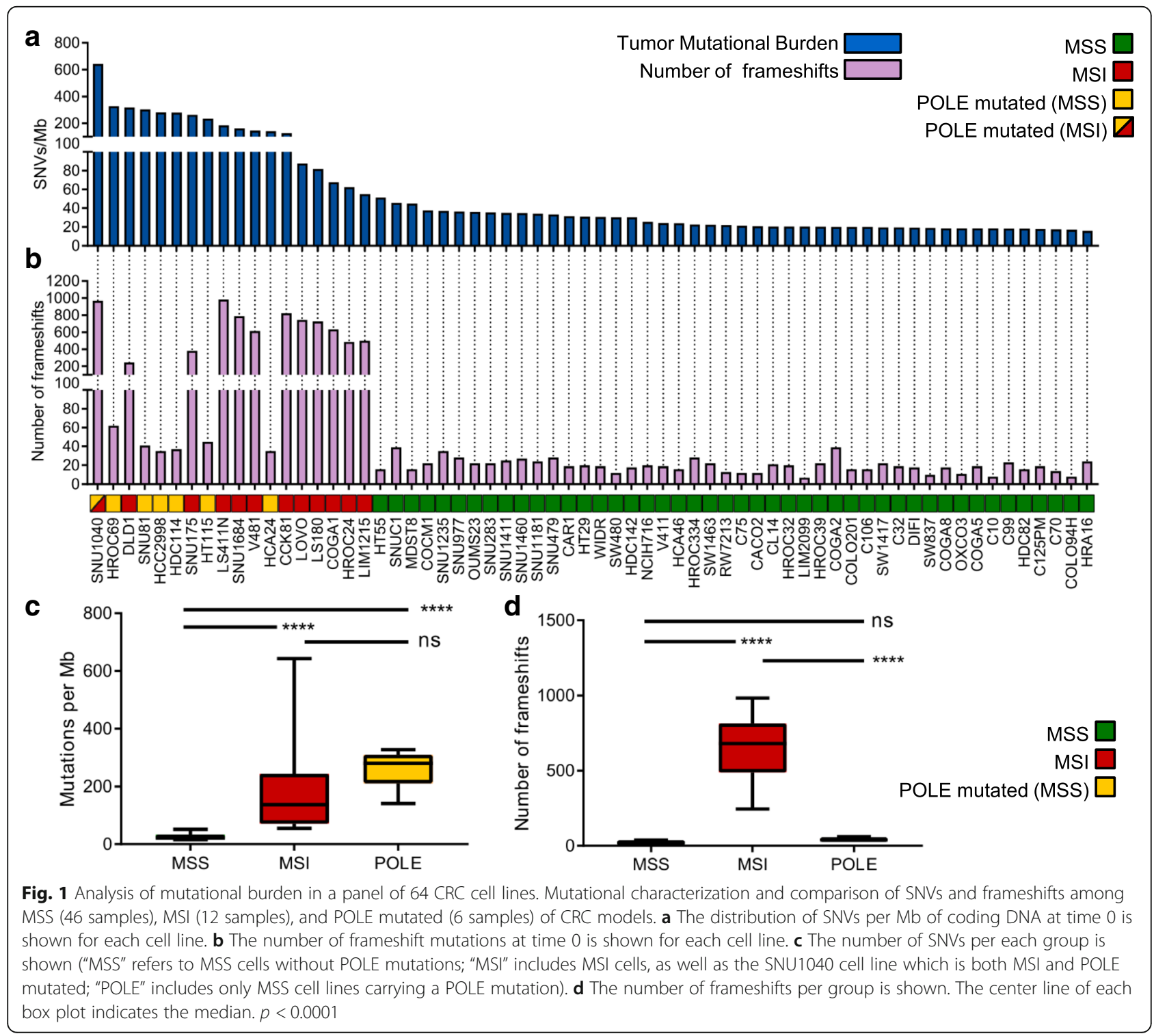


mice as described previously [24]. When reaching a volume of $1500-2000 \mathrm{~mm}^{3}$, the tumors were explanted, fragmented, and serially passaged in new mice. At each passage, part of the material was frozen for molecular analyses. Samples' genetic identity was determined by Sequenom-based analysis of 24 highly variable SNPs of germline DNA (Table 2), confirmed by analyzing preimplantation tumor material, and then validated every second passage in mice. The study population consisted of matched tumor and normal samples from 3 CRC patients that underwent surgical resection of liver metastases at the Candiolo Cancer Institute (Candiolo, Torino, Italy) and at the Mauriziano Umberto I Hospital (Torino) between 2009 and 2013. Patients signed informed consent, and the study was approved by the relevant institutional Ethics Committees.

\section{Western blotting analysis}

Proteins were extracted by solubilizing the cells in boiling SDS buffer (50 mM Tris- $\mathrm{HCl}$ [pH 7.5], $150 \mathrm{mM} \mathrm{NaCl}$, and $1 \%$ SDS). Samples were boiled for $5 \mathrm{~min}$ at $95^{\circ} \mathrm{C}$ and sonicated for $10 \mathrm{~s}$. Extracts were clarified by centrifugation, normalized with the BCA Protein Assay Reagent kit
(Thermo). Equal amounts of proteins $(20 \mu \mathrm{g})$ were loaded in each lane. Proteins were separated by PAGE and transferred to nitrocellulose sheets. Western blot detection was performed with enhanced chemiluminescence system (GE Healthcare) and peroxidase-conjugated secondary antibodies (Amersham). The following primary antibodies were used for western blotting: anti-beta2 Microglobulin [EP2978Y] (ab75853, Abcam), anti-MLH1 (ab92312, Abcam), anti-MSH2 (ab70270, Abcam), anti-MSH6 [EPR3945] (ab92471, Abcam), anti-MSH3 PA527864, Invitrogen, anti-PMS2 EPR3947 (Cell Marque Corporation, USA), anti-actin (I-19) (sc1616, Santa Cruz), and anti-HSP 90 $\alpha / \beta$ (H-114, sc-7947, Santa Cruz). Images were acquired with Chemidoc (Biorad), and western blot band intensity was analyzed using Image Lab software (Biorad).

\section{Results}

We selected from our database 64 CRC cell lines designed to recapitulate clinically relevant characteristics of CRC patients (Table 1 and Additional file 1: Figure S1a). Whole exome sequencing and RNAseq were performed on all models. Using previously developed computational tools

Table 2 List of SNPs used to identify patient-derived xenografts

\begin{tabular}{|c|c|c|c|c|}
\hline Probe & SNP & GENE & $\mathrm{Chr}$ & Functional consequence \\
\hline \multicolumn{5}{|l|}{ AMG_mid100 } \\
\hline rs11017876 & [A\G] & DOCK1 & 10:127402700 & Intron variant \\
\hline rs1106334 & {$[\mathrm{CAT}]$} & & 8:70100576 & \\
\hline rs1155741 & {$[\mathrm{C} T]$} & ITGA9 & 3:37585621 & Intron variant \\
\hline rs11655512 & {$[\mathrm{AlG}]$} & LOC339260 & 17:20948422 & Intron variant \\
\hline rs11940551 & {$[\mathrm{G} \backslash \mathrm{T}$} & & 4:27160856 & \\
\hline rs1210110 & {$[A \backslash G]$} & PRDM2 & 1:13770326 & Intron variant \\
\hline rs1364054 & {$[\mathrm{C} \backslash]$} & LINC00299 & $2: 8038605$ & Intron variant \\
\hline rs1528601 & {$[C \backslash G]$} & & 16:51064516 & \\
\hline rs161792 & {$[\mathrm{AlT}]$} & LOC101928166 & 3:152181915 & Intron variant \\
\hline rs 17272796 & [C।T] & PLCL2 & 3:17035776 & Intron variant \\
\hline rs 242076 & {$[\mathrm{CAT}]$} & SYN3 - TIMP3 & 22:32833844 & Intron variant \\
\hline rs4775699 & {$[\mathrm{CAT}]$} & SEMA6D & 15:47581352 & Intron variant \\
\hline rs4793172 & [AIT] & DCAKD & 17:45054112 & Intron variant \\
\hline rs4905366 & {$[A \backslash G]$} & & 14:95636762 & \\
\hline rs6603251 & {$[\mathrm{CAT}]$} & PPP2R3B & Y:359845 & Intron variant \\
\hline rs6734275 & {$[A \backslash G]$} & LOC105374785 & $2: 67014042$ & Intron variant \\
\hline rs685449 & {$[\mathrm{AIT}]$} & RGS17 & 6:153023396 & Intron variant \\
\hline rs7555566 & {$[A \backslash G]$} & KAZN & $1: 14478378$ & Intron variant \\
\hline rs7584993 & [A\C] & & 2:222981224 & \\
\hline rs7808249 & {$[A \backslash G]$} & CROT & 7:87354399 & Intron variant \\
\hline rs9293511 & {$[C \backslash T]$} & LOC105379072 & $5: 89120537$ & Intron variant \\
\hline rs9352613 & [A\G] & & $6: 78714716$ & \\
\hline rs9572094 & [C।T] & LOC105370159 & 13:34678745 & Intron variant \\
\hline
\end{tabular}




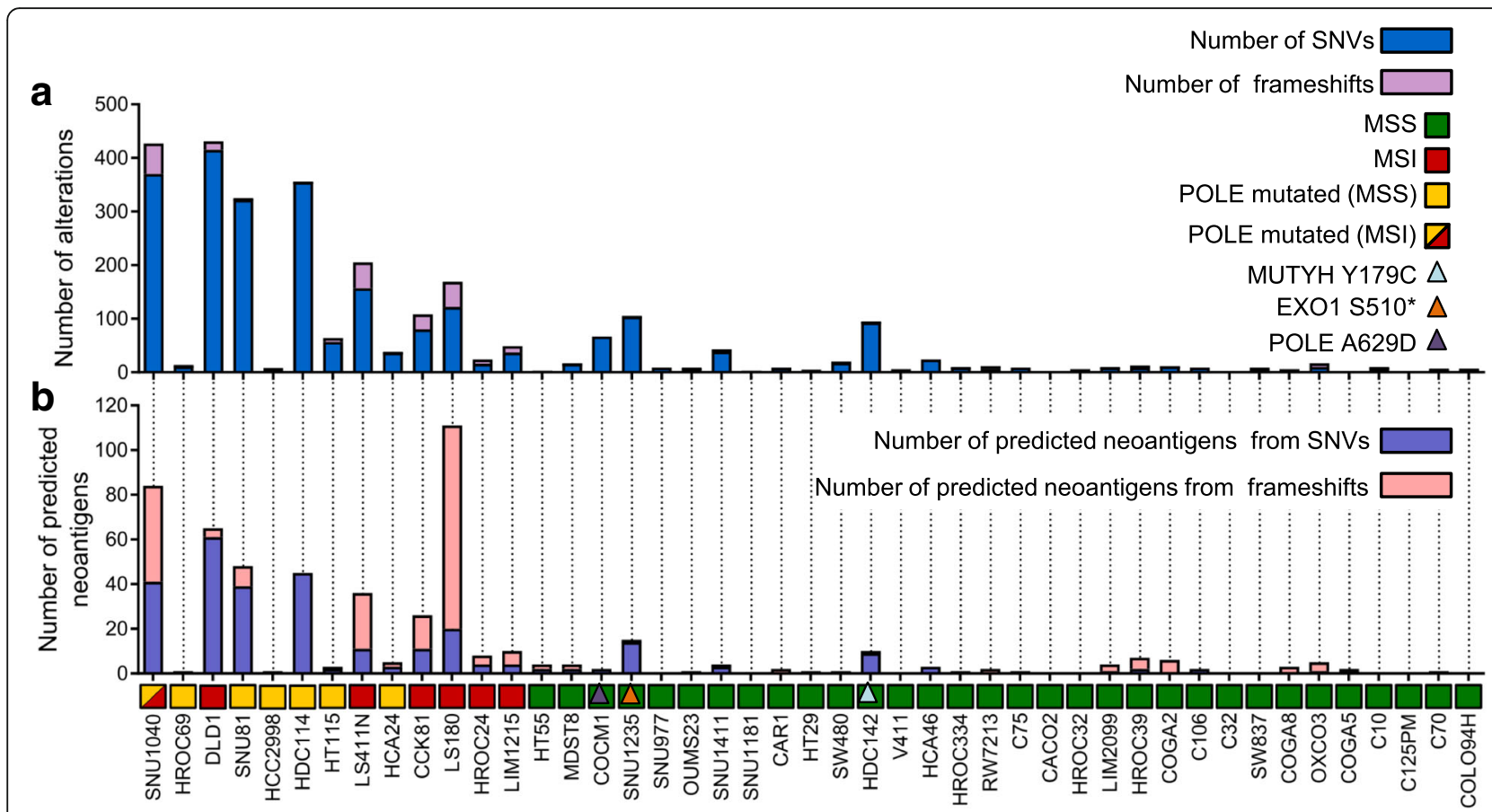

Fig. 2 In vitro evolution of mutational landscape in 45 CRC cell lines. Mutational characterization of CRC cells after 90 days of culture (T90) in vitro. a Bar charts show the number of novel alterations (SNVs and frameshifts) acquired at T90 (not present at T0) for each cell line. $\mathbf{b}$ The number of predicted neoantigens (see the "Methods" section) is shown. Each bar represents putative neoepitopes derived from SNVs and frameshifts

and bioinformatic algorithms $[13,14,25,26]$, we measured mutational burden (alterations per $\mathrm{Mb}$ ) assessing both SNVs and frameshifts (Fig. 1a, b, Additional file 2). Scrutiny of genomic alterations highlighted that MSI cell lines and those carrying known POLE hotspot mutations had higher number of mutations per $\mathrm{Mb}$ as compared to MSS cell lines (Fig. 1a). The type of DNA repair alterations occurring in each model affected the nature of mutations: MSI cells displayed a higher number of frameshifts and indels than POLE mutant cell lines; the opposite was true for SNVs (Fig. 1c, d).

Alterations in MMR and POLE genes are listed in Table 3 and Additional file 1: Figure S1b. The cell line with the highest number of variants (SNU1040) carried inactivating alterations in both $M L H 1$ and POLE (Additional file 1: Figure S1b). Altogether, these results are consistent with what has been reported in CRC patients carrying alterations in the MMR DNA repair pathway, indicating that the cell models included in this study broadly recapitulate what is observed in clinical specimens [27].

To assess whether, and to what extent the basal mutational profiles (Time 0: T0) evolved over time, we passaged 45 cell lines for 90 days and collected a second set of samples (Time 90: T90) (Additional file 1: Figure S2). These were subjected to WES and analyzed using the computational pipeline described above. Across all cell lines globally, the total mutational burden was similar between T0 and T90 (Additional file 1: Figure S3). However, when the T0 and T90 mutational profiles were compared, prominent differences were detected among models sharing specific DNA repair defects (Fig. 2a). Specifically, the mutational landscapes of most MSI and POLE mutant cells evolved very rapidly through the generation of novel SNVs and frameshifts (Fig. 2a). On the contrary, the majority of MSS models showed more stable profiles (Fig. 2a). We sought to minimize confounding effects due to differences in cell-intrinsic doubling times (Table 1); we therefore calculated the doubling time of all cell models (Table 1, Additional file 1: Figure S4). Notably, evolvability trends remained apparent after normalization for doubling time (Additional file 1: Figure S5). We designated rapidly evolving CRC cells as EVOLVING-CRC and evolutionary stable CRC cell as STABLE-CRC (Table 1).

We empirically define EVOLVING-CRCs as those cells that acquire 10 alterations (or more) per day after normalizing mutation data to the doubling time of cell lines (Table 1). Moreover, EVOLVING-CRCs often carried alterations in multiple genes involved in distinct DNA repair functions, suggesting that defects in several DNA damage response pathways might be co-selected (Additional file 1: Figure S1b). The expression of MMR genes was assessed by western blot at T0 and 


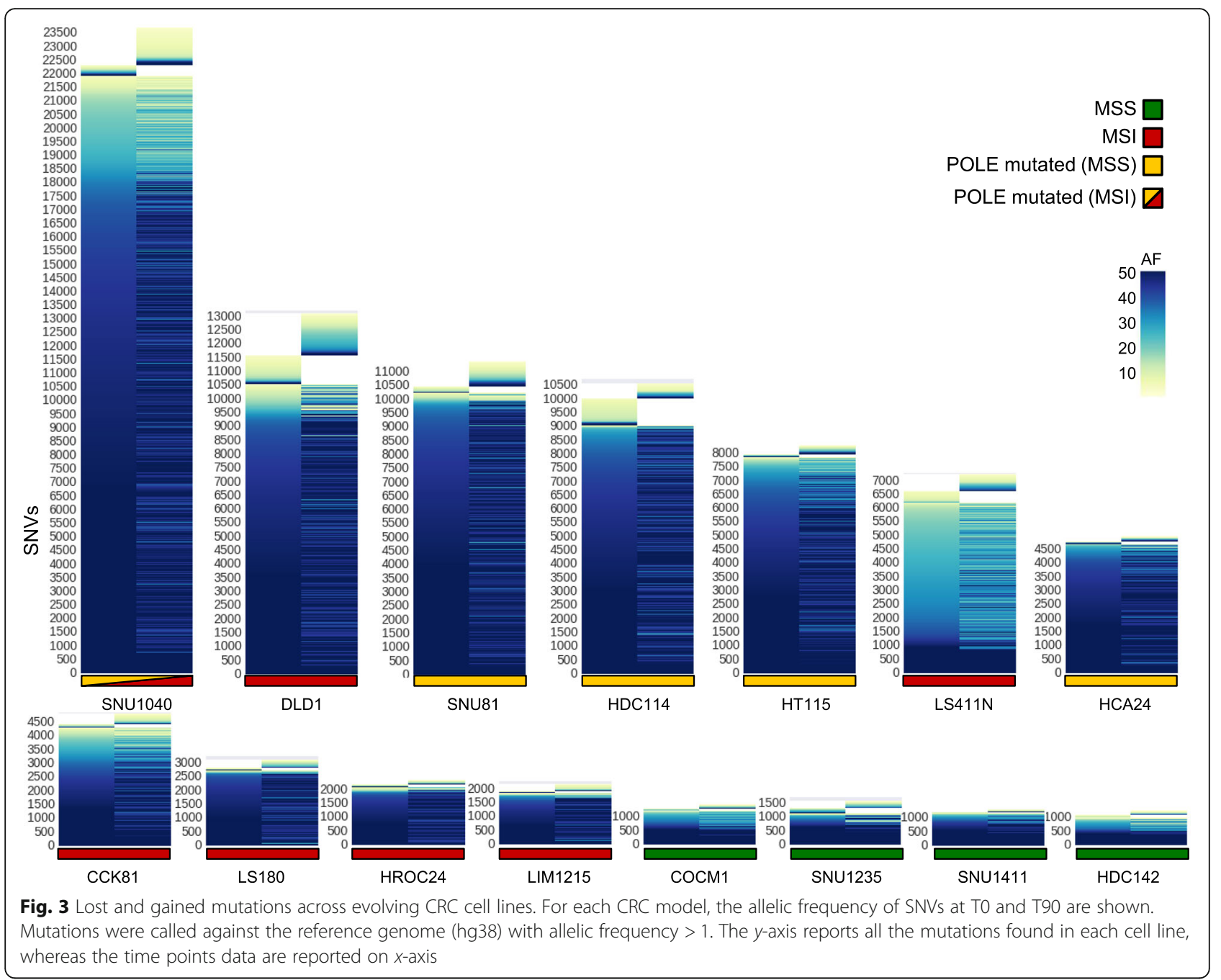

T90, and no differences were observed (Additional file 1: Figure S6).

The genome of four CRC lines classified as MSS (SNU1235, COCM1, HDC142, and SNU1411) exhibited dynamic mutational profiles (Fig. 2). In an attempt to decipher the molecular basis of these findings, whole exome data of the outliers were carefully examined, focusing on genes previously implicated in DNA repair pathways that are not routinely subjected to scrutiny in CRC patients. We found that SNU1235 and HDC142 models carried biallelic alterations in the EXO1 (S510*) and MUTYH $(\mathrm{S} 179 \mathrm{C})$ genes, respectively. The exonuclease EXO1 is implicated in both MMR (it binds MLH1) and base excision repair [28], while MUTYH encodes a DNA glycosylase that is involved in oxidative DNA damage repair and is part of the base excision repair pathway [29]. Germline mutations in MUTYH cause MUTYH-associated polyposis (MAP) [30]. Scrutiny of the COCM1 exome revealed a POLE variant (A629D). A629 is localized in a region of POLE highly conserved during evolution (Additional file 1: Figure S7). The A629D change is potentially damaging according to the SIFT [31] and Polyphen [32] algorithms, which predict the putative impact of amino acid substitutions on human proteins using structural and comparative evolutionary considerations.

We next addressed how longitudinal evolution of CRC cell genomes affected their predicted neoantigen profile. To this end, WES, RNAseq, and HLA prediction data were combined as previously described [9]. In detail, we identified genomic variants that satisfied three criteria: (i) emerged over time, (ii) occurred in transcribed genes, and (iii) scored positively when HLA I matching algorithms were applied. The variants that emerged after deploying the above computational pipeline were classified as putative neoantigens (Fig. 2b). Hypermutated and EVOLVINGCRC cells displayed higher levels of putative neoantigens compared to slowly evolving CRC cells (Fig. 2b). Moreover, and consistent with their predicted effects on antigenicity, a high prevalence of indels and associated frameshifts, 


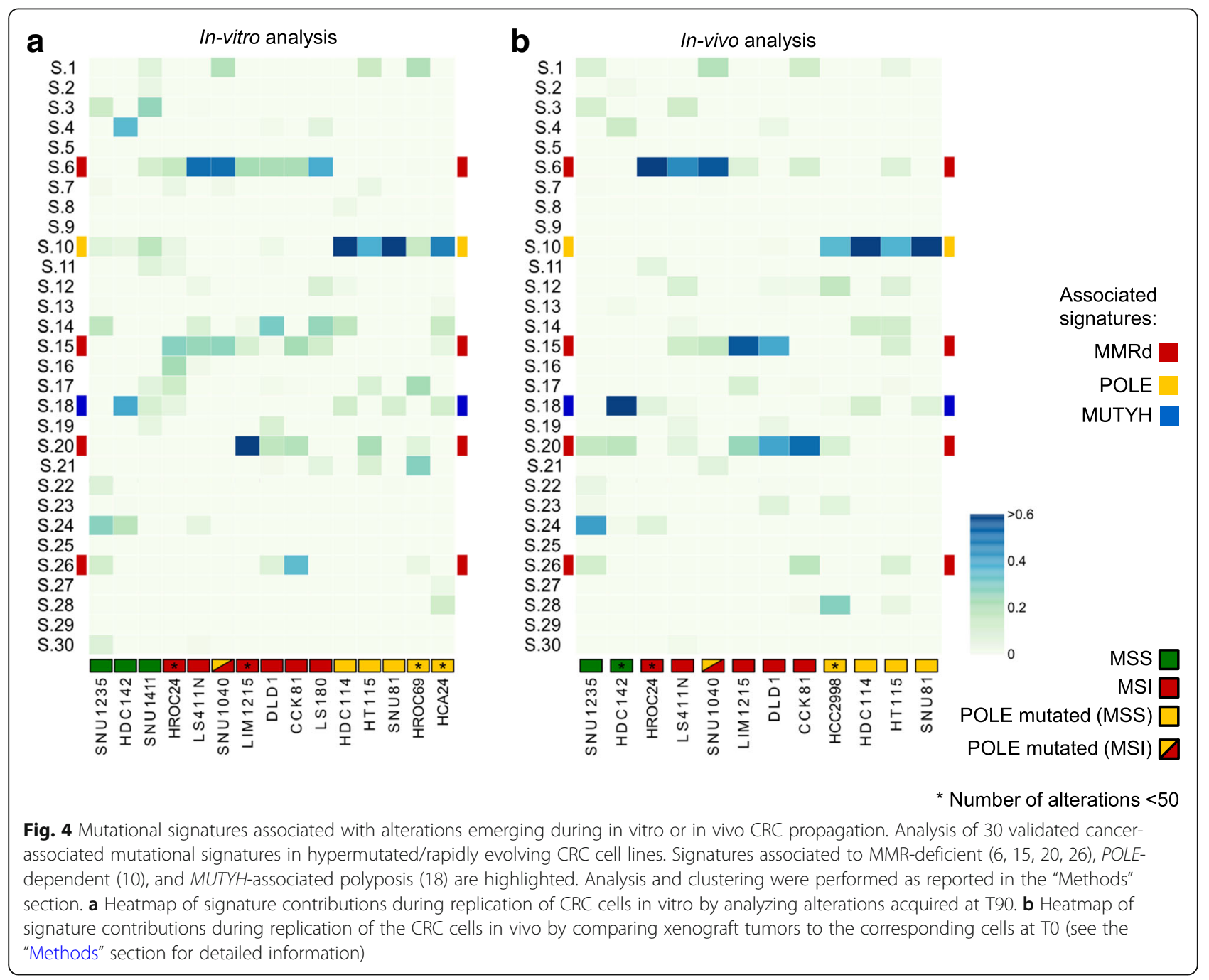

which occur in MSI CRCs, translated into higher numbers of predicted neoantigens in this subset (Fig. 2b).

Next, we studied whether in parallel to mutation gains we could also detect loss of variants over time. For this reason, we tracked lost and gained alteration in "evolving" cell lines over time. As expected, variants that did not change over time showed high allelic frequency, likely reflecting their clonal (trunk) status. Mutations that emerged or were lost showed lower allelic frequency (Fig. 3).

Mutational signatures are characteristic combinations of mutation types arising from mutagenesis processes such as alterations in DNA replication, exposure to DNA damaging agents, tissue culture conditions, and DNA enzymatic editing [18]. In human tumors, over 30 mutational signatures have been identified, a subset of which are linked to defective DNA repair pathways. For example, signatures 6, 15, 20 , and 26 are associated with MMR defects and signature 10 is linked to inactivating mutation in the proofreading domain of DNA polymerases, while signature 18 appears to explain the rise of 8-oxoG:A mismatches due to $M U T Y H$ biallelic alteration [33].

We reasoned that the remarkable evolvability observed in a subset of CRC cells might be reflected in their mutational signatures. To test this, we first identified mutational signatures at T0. As expected, MSI cells displayed signatures $6,15,20$, and 26 , while POLE mutant cells showed primarily mutational signature 10 (Additional file 1: Figure S8).

We next assessed which signatures were acquired (remained active) during replication of the cells in vitro by comparing samples collected at $\mathrm{T} 0$ and $\mathrm{T} 90$. We found that in most instances, DNA alterations linked to MMR and POLE defects continued to occur over time, indicating that the corresponding DNA repair capabilities were permanently disabled (Fig. 4a).

Replication of cancer cell populations in 2D is thought to encounter little or no selective pressure as the cells are 
Table 3 POLE mutations in CRC cells

\begin{tabular}{|c|c|c|c|c|c|c|c|c|}
\hline \multicolumn{9}{|c|}{ Time point 0} \\
\hline \multirow[b]{2}{*}{ SAMPLE } & \multirow[b]{2}{*}{ Coord (HG38) } & \multirow[b]{2}{*}{$\begin{array}{c}\text { AA } \\
\text { Change }\end{array}$} & \multicolumn{3}{|c|}{ DNA } & \multicolumn{3}{|c|}{ RNA } \\
\hline & & & $\begin{array}{c}\text { Normal } \\
\text { allele } \\
\text { counts }\end{array}$ & $\begin{array}{l}\text { Altered } \\
\text { allele } \\
\text { counts }\end{array}$ & $\begin{array}{c}\text { Allelic } \\
\text { Frequency }\end{array}$ & $\begin{array}{c}\text { Normal } \\
\text { allele } \\
\text { counts }\end{array}$ & $\begin{array}{l}\text { Altered } \\
\text { allele } \\
\text { counts }\end{array}$ & $\begin{array}{c}\text { Allelic } \\
\text { Frequency }\end{array}$ \\
\hline HCA24 & chr12:132673261 & p.S459F & 33 & 26 & $44.07 \%$ & 9 & 9 & $50.00 \%$ \\
\hline HCC2998 & chr12:132676598 & p.P286R & 54 & 44 & $44.90 \%$ & 27 & 25 & $48.08 \%$ \\
\hline HDC114 & chr12:132673271 & p.A456P & 33 & 36 & $52.17 \%$ & 17 & 28 & $62.22 \%$ \\
\hline HROC69 & chr12:132673703 & p.V411L & 88 & 64 & $42.11 \%$ & 31 & 16 & $34.04 \%$ \\
\hline HT115 & chr12:132673703 & p.V411L & 100 & 62 & $38.27 \%$ & 24 & 10 & $29.41 \%$ \\
\hline SNU1040 & chr12:132675740 & p.F367L & 34 & 16 & $32.00 \%$ & 25 & 37 & $59.68 \%$ \\
\hline SNU81 & chr12:132676598 & p.P286R & 40 & 30 & $42.86 \%$ & 22 & 26 & $54.17 \%$ \\
\hline \multicolumn{9}{|c|}{ Time point 90} \\
\hline \multirow[b]{2}{*}{ SAMPLE } & \multirow[b]{2}{*}{ Coord (HG38) } & \multirow[b]{2}{*}{$\begin{array}{c}\text { AA } \\
\text { Change }\end{array}$} & \multicolumn{3}{|c|}{ DNA } & \multicolumn{3}{|c|}{ RNA } \\
\hline & & & $\begin{array}{c}\text { Normal } \\
\text { allele } \\
\text { counts }\end{array}$ & $\begin{array}{l}\text { Altered } \\
\text { allele } \\
\text { counts }\end{array}$ & $\begin{array}{c}\text { Allelic } \\
\text { Frequency }\end{array}$ & $\begin{array}{c}\text { Normal } \\
\text { allele } \\
\text { counts }\end{array}$ & $\begin{array}{l}\text { Altered } \\
\text { allele } \\
\text { counts }\end{array}$ & $\begin{array}{c}\text { Allelic } \\
\text { Frequency }\end{array}$ \\
\hline $\mathrm{HCA} 24$ & chr12:132673261 & p.S459F & 33 & 27 & $45.00 \%$ & 17 & 20 & $54.05 \%$ \\
\hline HCC2998 & chr12:132676598 & p.P286R & 37 & 32 & $46.38 \%$ & 11 & 17 & $60.71 \%$ \\
\hline HDC114 & chr12:132673271 & p.A456P & 37 & 20 & $35.09 \%$ & 30 & 37 & $55.22 \%$ \\
\hline HROC69 & chr12:132673703 & p.V411L & 83 & 44 & $34.65 \%$ & 49 & 23 & $31.94 \%$ \\
\hline HT115 & chr12:132673703 & p.V411L & 146 & 38 & $20.65 \%$ & 18 & 4 & $18.18 \%$ \\
\hline SNU1040 & chr12:132675740 & p.F367L & 24 & 17 & $41.46 \%$ & 11 & 33 & $75.00 \%$ \\
\hline SNU81 & chr12:132676598 & p.P286R & 65 & 56 & $46.28 \%$ & 15 & 22 & $59.46 \%$ \\
\hline
\end{tabular}

MSS

MSI

cultured in the same conditions for many generations before the experiment is started. To monitor mutational and neoantigen evolution under more stressful (selective) conditions, CRC cells including MSS, MSI, and POLE models were transplanted in immunodeficient (NOD SCID) mice and allowed to grow until they reached approximately $1000 \mathrm{~mm}^{3}$ in size, after which tumors were excised. Although NOD SCID mice have no adaptive immunity, the mouse stromal microenvironment and elements of cellular innate immunity are known to affect the growth of human cancer cells in vivo [34]. DNA samples were obtained before implantation and at the end of the experiment. WES was performed, and the data were analyzed with the same bioinformatic pipeline applied to cells grown in vitro. The mutational profiles revealed higher evolutionary rates in vivo than in vitro (Additional file 1: Figure S9a, b). This translated into increased levels of predicted neoantigens in vivo (Additional file 1: Figure S9c). Notably, mutational signatures linked to MSI status and POLE mutations were more marked in vivo than in vitro (Fig. $4 \mathrm{~b}$, Additional file 1: Figure S10). We then assessed whether the mouse microenvironment exerts selection on the cells expanded in vivo and compared the results to cells passaged in vitro. To this end, we characterized the ratio between non-synonymous and synonymous mutations in vitro and in vivo. We detected very limited or no selection in cells passaged in vitro (ratio 3:1). Instead, in vivo the ratios for lost and gained mutations were 1:1 and 2:1, respectively, indicating a purifying selection (Additional file 1: Figure S11). These findings suggest that when cells are transplanted in mice they are subjected to environmental selection.

Next, we asked whether the evolutionary trajectories observed in CRC cells with alterations in DNA repair pathways also occurred in human CRC with analogous molecular profiles. To this end, we selected MMRproficient, MMR-deficient, and POLE mutant cases (Table 4) from our extensive patient-derived CRC xenograft biobank [35]. Each model was serially transplanted for at least four generations in immunodeficient mice as described in the phylogenetic tree (Fig. 5a). Samples collected at each transplantation were subjected to WES. In some instances, simultaneous transplantation of the 


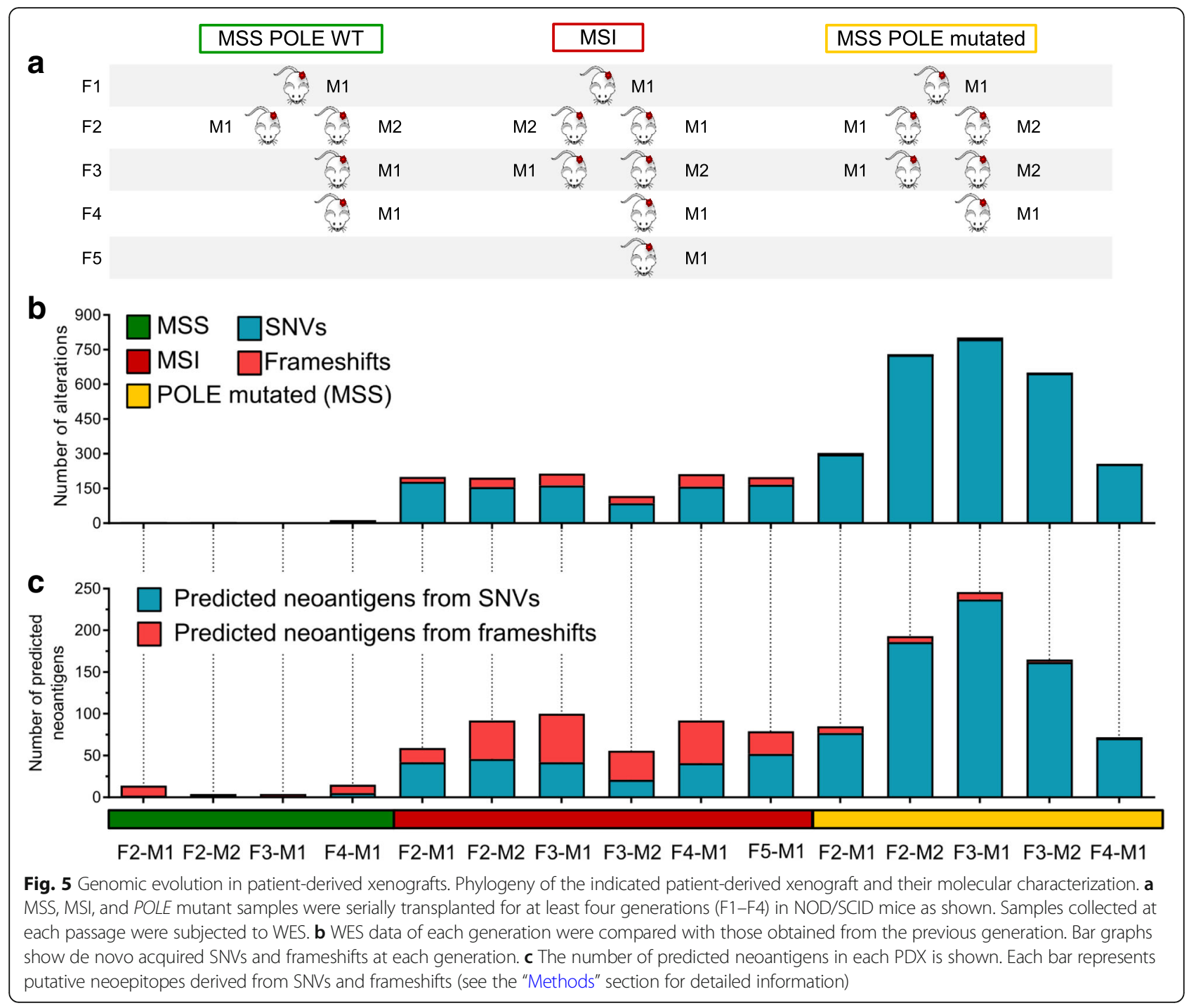

same tumor in two animals allowed acquisition of independent measurements for each generation. NGS data were analyzed with the bioinformatic pipeline applied to cells grown in vitro. These experiments revealed remarkable differences in the evolvability of MSS, MSI, and POLE CRC models in vivo and indicated that these characteristics also occurred in patient-derived CRC samples (Fig. 5b, c). As expected, high-frequency (clonal-trunk) variants were conserved across generations. Interestingly, the in vivo results differ from those obtained in cell models in vitro. We find that in PDX models, not only sub-clonal but also clonal populations can emerge in the subsequent generation of colorectal cancers with DNA repair defects (Fig. 6).

Furthermore, in MSI and POLE patient-derived xenografts, the mutational signatures were continuously (re) generated and could be clearly recognized (Additional file 1: Figures S12 and S13). In non-mutator (slow evolving) cell lines, very few mutations emerged over time, and thus, the possibility to assess mutational signatures was limited. Because of this, in the slowly evolving models, we were unable to reliably generate mutational signatures.

Distinct subsets of CRCs can be recognized based on histological characteristics, as well as their genomic, epigenetic, and transcriptional profiles. As a result, CRC can be classified into specific subsets, which are often correlated with divergent clinical outcomes [36, 37]. The rate of genomic evolution and the dynamics of neoantigen profile have not yet been systematically explored as a method to classify CRC. We therefore asked whether any molecular traits (beyond alterations in DNA repair genes) could distinguish EVOLVING-CRC and STABLE-CRC. To address this question, we performed unbiased gene copy number and transcriptional comparative analyses of CRC cell lines. As previously reported, MSI CRC cells 


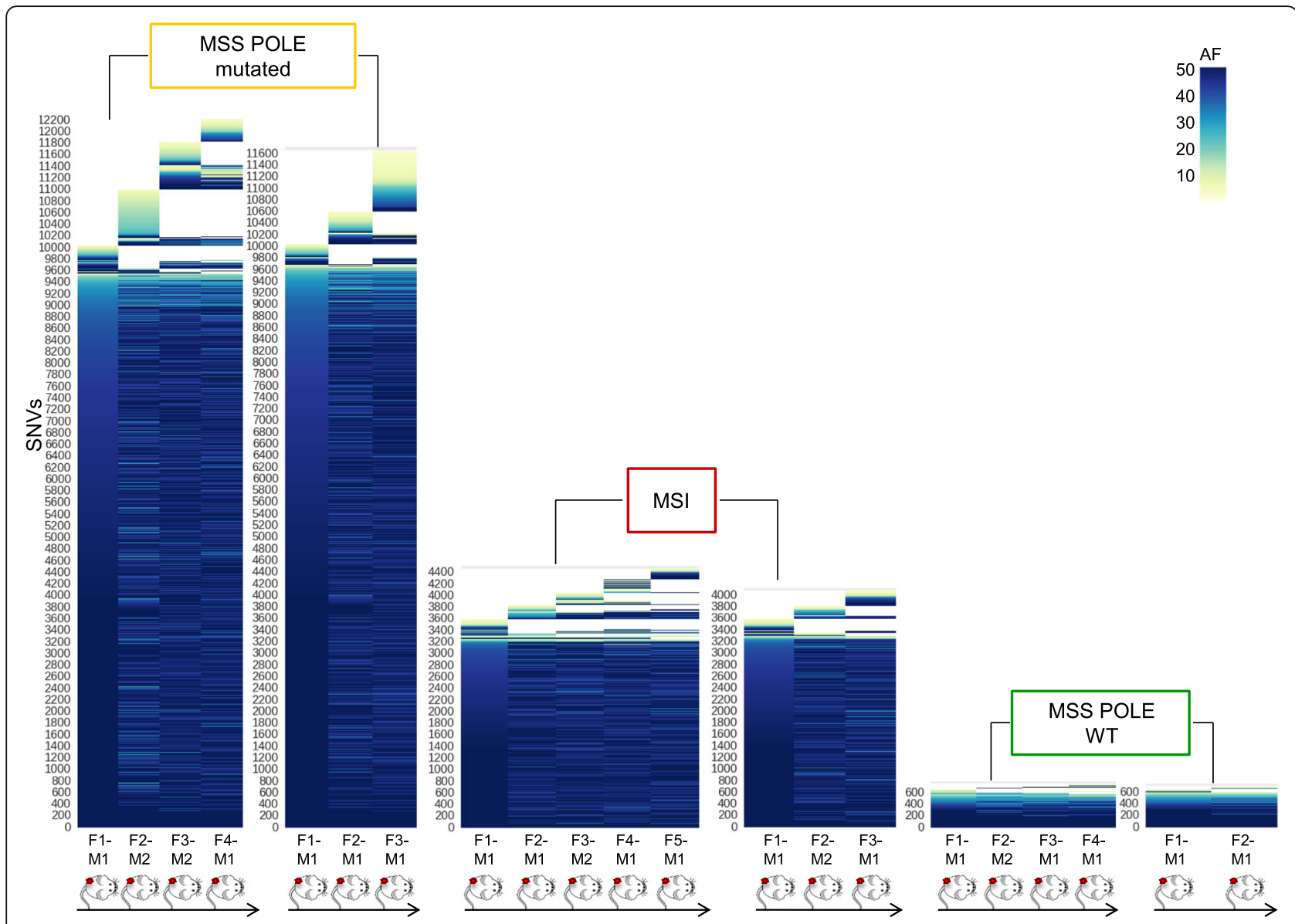

Fig. 6 Lost and gained mutations across the indicated PDX generations. The color code defines allelic frequencies of acquired SNVs at each generation (with allelic frequency $>1$ ). The $y$-axis lists all SNVs identified in each branch; the mouse generation (genealogy) is reported on the $x$-axis

typically carried a close to diploid chromosomal status, while MSS showed elevated aneuploidy (Fig. 7) [38]. Interestingly, the most rapidly evolving POLE mutant lines, SNU81 and HDC114, also displayed a diploid prevalent phenotype. Nonetheless, copy number and ploidy status could not distinguish "EVOLVING" and "STABLE" CRC models.

Next, we performed RNAseq on the entire dataset to explore whether transcriptional profiles could classify rapidly evolving CRC lines. Differential analysis of RNAseq data was initially performed comparing the MSS and MSI sample groups. The list of differentially expressed genes was consistent to results previously reported in this setting, and 168 genes were differentially expressed between these two groups (Table 5) [39]. Next, we evaluated genes differentially expressed in hypermutated versus non-hypermutated cells, grouping together MSI- and POLE-mutated cell lines and comparing them to the MSS lines (Fig. 8a). Notably, proteins associated with immune response and predominantly with antigen-presenting and antigen recognition functions were consistently downregulated in cell lines with high mutational burden (Fig. 8b). Next, we compared EVOLVING and STABLE CRC models. The number of genes differentially expressed with significant $p$ value was smaller due to the reduced number of available samples (Fig. 9a). Beta-2 microglobulin (B2M) was downregulated in most EVOLVING as compared to STABLE CRCs (Fig. 9b, c). Downregulation of B2M was confirmed at the protein level (Fig. 9c) and was frequently associated with premature stop codons in the $B 2 M$ gene (Fig. 9d). Interestingly, the four MSS models (COCM1, SNU1235, SNU1411, and HDC142) with low mutational burden but dynamic mutational profile also displayed low levels of B2M (Fig.9b, c). Comparison of EVOLVING and STABLE CRC models pinpointed other genes differentially expressed including CPNE1, IRF1, and PMSB10. These genes are also involved in immune-related processes and their downregulation might similarly reduce immune surveillance of EVOLVING CRCs (Fig. 9a and Additional file 1: Figure S14). We next performed the analysis showed in Fig. 9a in a multivariate fashion 


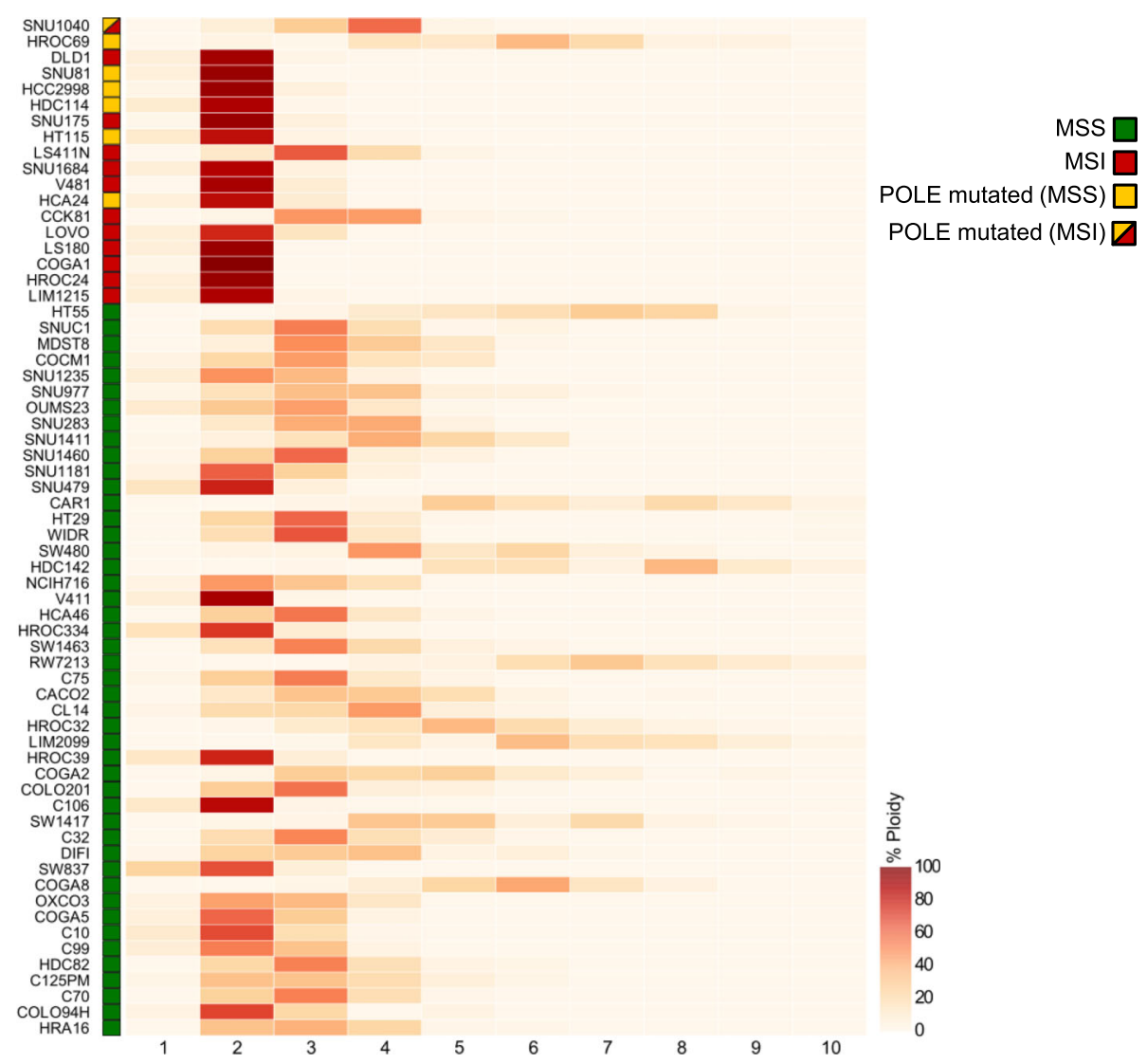

Fig. 7 Analysis of cell ploidy in a panel of 64 CRC cell lines. Heatmap showing distribution of ploidy for every segmented region in each cell line. Samples are sorted from most to less mutated as reported in Fig. 1. The percentage (ploidy) is calculated as described in detail in the "Methods" section

taking into account the growth rates of the cells or the number of mutations normalized to the doubling time. The number of statistically significant genes in the multivariate analyses (Additional file 1: Figure S15) was lower but consistent with the findings of Fig. 9a. In the future, it would be interesting to assess whether the differential expression of genes in fast evolving CRC models has a functional impact. This aspect cannot be causally predicted at this stage.

\section{Discussion}

In the past decade, it has become clear that most human tumors are highly molecularly heterogeneous, and this affects prognosis and the emergence of therapeutic resistance [40]. How tumor-specific somatic variations can lead to distinct neoantigen profiles and ultimately to immune surveillance has also been partially elucidated. The number of neoantigens depends on several factors. For example, lung cancers associated with smoking habits have high levels of mutations [41, 42], whereas the development of skin melanomas is correlated with UV lightmediated mutagenicity [43]. Both smoking and UV exposure occur during defined periods and their mutagenicity is transient, leading to high-but relatively stable-mutational profiles [44, 45]. Another class of tumors with high mutational burden is characterized not by exposure to external carcinogens, but rather by the intrinsic inability of tumor cells to efficiently repair DNA. The latter is due to epigenetic or genetic alterations in key effectors of

Table 4 Molecular characterization of patient-derived xenografts

\begin{tabular}{lllllllllll}
\hline Sample & MSI status & BRAF & KRAS & NRAS & MLH1 & MSH2 & MSH3 & MSH6 & PMS2 & POLE \\
\hline CRC106 & MSI & V600E & WT & WT & WT & WT & WT & R577H / p.T1085Tf57* & WT & WT \\
CRC542 & MSS & WT & WT & WT & WT & WT & WT & WT & WT & WT \\
CRC371 & MSS & WT & WT & E132K & R265C & WT & WT & E946* & WT & V411L
\end{tabular}

*Only non-synonymous variants present in COSMIC database are reported 


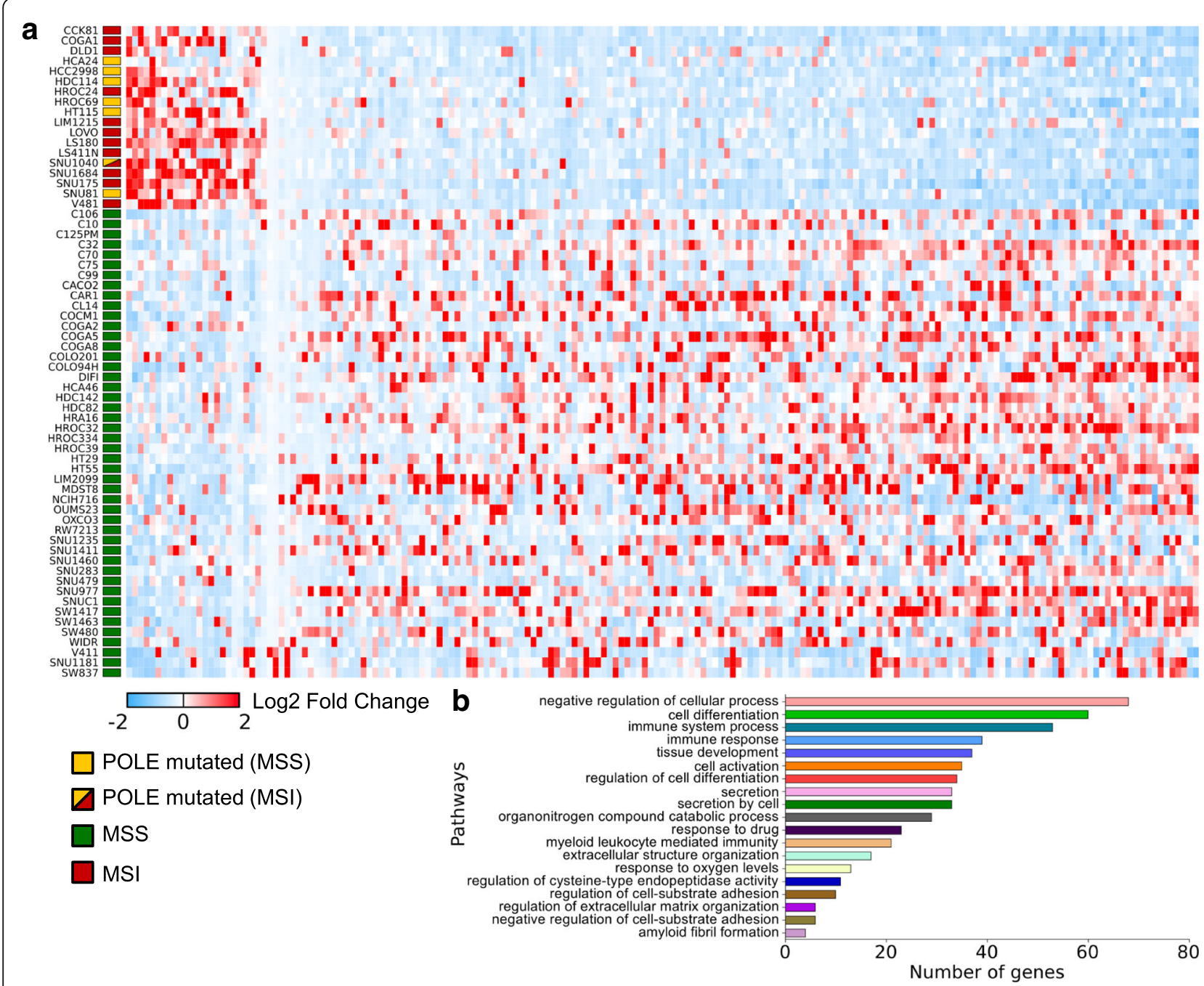

Fig. 8 Transcriptional analysis of CRC cell lines. Differential expression analysis between hypermutated and non-hypermutated cells. a 183 unique genes differentially expressed between hypermutated (MSI/POLE) versus non-hypermutated CRC cells (MSS). Log2 expression values along with the mean change in expression are shown. $\mathbf{b}$ Pathway analysis of genes differentially expressed between hypermutated versus non-hypermutated CRC cells using g:Profiler application (see the "Methods" section)

DNA repair pathways, rather than acute or chronic carcinogen exposure. In this work, we used CRC as a model system to understand whether and to what extent alterations of DNA repair pathway components modulate neoantigen profiles over time in vitro and in vivo. Tumors carrying alterations affecting DNA repair genes maintained their molecular characteristics over time, and in most instances, the functional consequence of those alterations is continuous and propagated at every generation. An exception was represented by two POLE mutant CRC cell lines (HROC69 and HCC2998) which despite having high mutational burden did not appreciably evolve over time. The reason(s) for this phenotype is presently unclear. Interestingly, these two POLE mutant cells that evolved poorly over time had less marked mutational signatures, possibly suggesting that, in these models, polymerase defects may undergo some form of functional compensation.

The longitudinal analysis of cell and PDX models highlighted several aspects. For example, MSI- and POLE-mutated tumors tended to acquire SNV or short insertions/deletions over time. These alterations can lead to novel putative neoantigens which potentially trigger the host immune system. In addition to well-known DDR genes (MLH1, MSH2, MSH6, PMS2, POLE), our study indicate that other genes involved in DNA repair pathway may lead to accumulations of mutations possibly translating in novel epitopes. EXO1 and MUTYH are two of such examples. Profiling of these genes in the clinical setting may help to intercept tumors not classified as unstable or with hypermutator phenotype but nevertheless continuously evolving and accumulating mutations. 

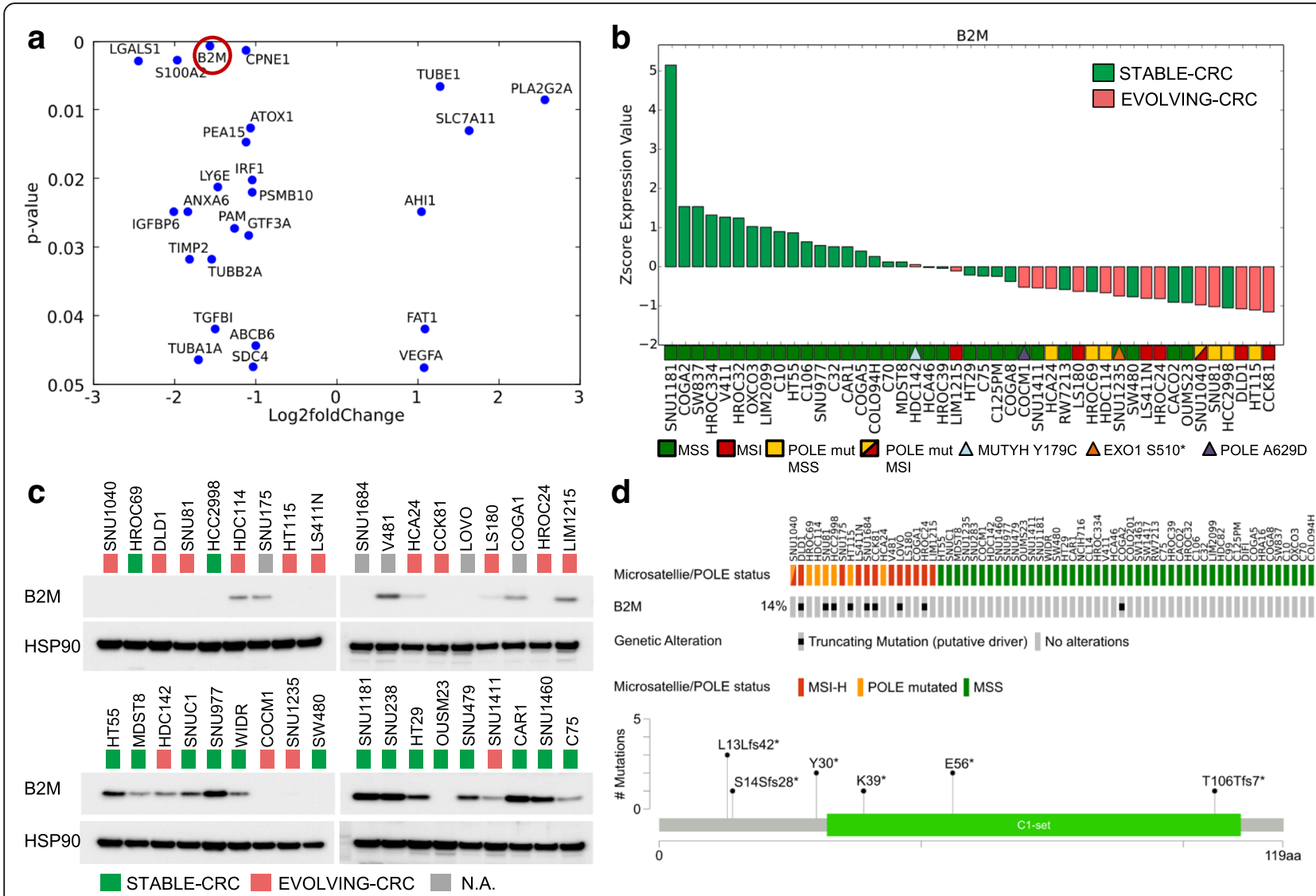

d

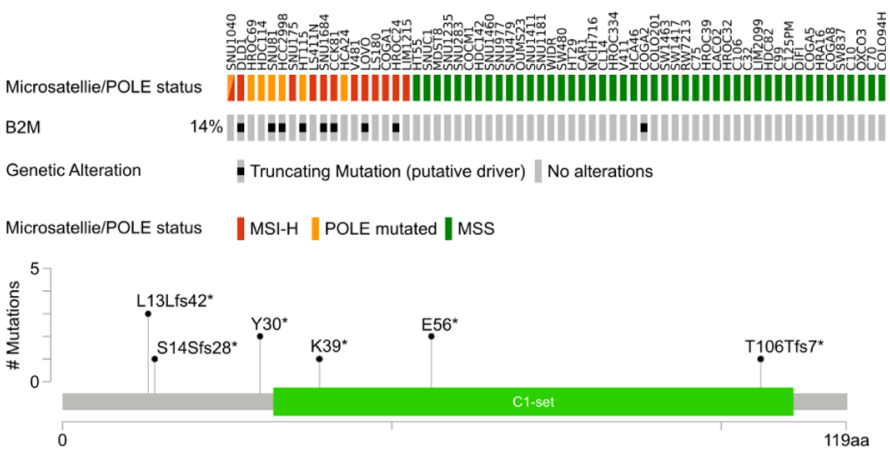

Fig. 9 Beta2 microglobulin (B2M) expression is downregulated in EVOLVING-CRC. Transcriptional and protein levels of the B2M gene. a Genes differentially expressed in EVOLVING-CRC relative to STABLE-CRC with a significant $p$ value $(p<0.05)$. $\mathbf{b}$ Waterfall chart showing B2M expression at RNA level across a panel of 45 CRC cell lines. c Western blot analysis of B2M expression. In gray are highlighted samples for which T90 sequencing were not available. Blots were reprobed with anti-HSP90 antibody to confirm equal loading. $\mathbf{d}$ B2M gene alterations on 64 CRC cell lines at T0 (upper panel) and codon affected (lower panel)

Our analysis suggests that in parallel to mutation gains, loss of variants also occurs during cell propagation. Our data indicate that in hypermutated CRCs, including MSIand POLE-mutated models expanded in vitro, these events are mainly confined to subclones. A limitation of this study is that longitudinal characterization of lost and gained mutations in vitro could be influenced by sampling of cell populations during cell passaging. We also report that in the propagation of PDXs, possibly due to selection imposed by the microenvironment, not only subclonal but also clonal variants emerge de novo over time. Based on these results, we speculate that in CRC patients with DNA repair defects metastatic seeding or therapeutic debulking can lead to the emergence of new subsets of clonal neoantigens. This could have implications for the development of therapies relying on the presence of clonal neoantigens, such as ICP, CAR-T, and vaccines.

Both cell lines and PDXs have been widely employed to test anticancer compounds [46-48]; however, experimental reproducibility has occasionally been questioned $[49,50]$. The molecular evolvability that we find to occur during serial passaging of cells and PDXs may partly account for the discrepant results obtained with these models [51-53].

A limitation of the present study is that it examined the evolution of cell lines and xenografts but cannot address the impact of the immune system in the evolutionary dynamics due to intrinsic limitations of the models we used.

Our data indicate that alterations in DNA repair genes facilitate the acquisition of neoantigens. These novel putative epitopes can be recognized by the immune system. Accordingly, we confirm that CRCs with high number of mutations (hypermutated CRCs) selectively downregulate components of the neoantigen presentation process, such as $B 2 M$, thus restricting the ability of the host immune system to detect them. Our results further suggest that non-hypermutated CRCs, that display fast evolving mutational and antigen profiles, also show downregulation of components implicated in neoantigen presentation. The differences in expression of molecules involved in immune functions we observed in the CRC models could have originated 
Table 5 List of genes differentially expressed in the indicated cell lines

\begin{tabular}{|c|c|c|c|c|c|}
\hline gene_id & Log2 fold change & $p$ adj. & gene_id & Log2 fold change & $p$ adj. \\
\hline MSS vs MSI & & & CYFIP2 & 1.37 & 0.03 \\
\hline ABCB6 & -1.12 & 0.01 & DAB2IP & -1.14 & 0.00 \\
\hline ACAD11 & 1.18 & 0.00 & DCBLD2 & -2.43 & 0.00 \\
\hline ACOT8 & -1.04 & 0.00 & $\mathrm{DD} 27$ & -1.02 & 0.00 \\
\hline AHCYL2 & 1.12 & 0.02 & DNTTIP1 & -1.25 & 0.00 \\
\hline ALDH6A1 & 1.34 & 0.00 & DPEP1 & -1.88 & 0.04 \\
\hline AMACR & -1.54 & 0.01 & DYNLRB1 & -1.04 & 0.00 \\
\hline $\mathrm{AOC1}$ & -1.57 & 0.04 & EDN1 & -1.79 & 0.01 \\
\hline APBB3 & 1.04 & 0.00 & EMP1 & -1.47 & 0.03 \\
\hline ARHGAP18 & -1.01 & 0.02 & EPB41L1 & -1.09 & 0.01 \\
\hline ATOX1 & -1.00 & 0.01 & EPB41L4A-AS1 & 1.04 & 0.01 \\
\hline ATP5E & -1.22 & 0.00 & EPS8L1 & -1.13 & 0.03 \\
\hline ATP9A & -1.77 & 0.00 & FCGRT & -1.69 & 0.00 \\
\hline B2M & -1.58 & 0.00 & $\mathrm{FECH}$ & 1.07 & 0.00 \\
\hline B4GALT5 & -1.14 & 0.00 & FKBP1A & -1.02 & 0.01 \\
\hline BCL2L15 & -1.73 & 0.01 & FZD7 & -1.02 & 0.03 \\
\hline BCL9L & -1.09 & 0.02 & GABBR1 & 1.70 & 0.01 \\
\hline BPTFP1 & 1.09 & 0.03 & GABRE & -1.83 & 0.00 \\
\hline C15orf52 & -1.28 & 0.05 & GCAT & 1.39 & 0.00 \\
\hline C20orf24 & -1.14 & 0.00 & GLS2 & 1.38 & 0.00 \\
\hline C6orf48 & 1.05 & 0.01 & GNE & -1.13 & 0.04 \\
\hline CAPG & -1.30 & 0.02 & GSN & -1.30 & 0.03 \\
\hline CCDC71L & -1.08 & 0.02 & HELZ2 & -1.14 & 0.01 \\
\hline CCND3 & -1.26 & 0.00 & $\mathrm{HIST} 1 \mathrm{H} 2 \mathrm{AC}$ & -1.36 & 0.01 \\
\hline CCPG1 & 1.16 & 0.02 & $\mathrm{HIST} 1 \mathrm{H} 2 \mathrm{BD}$ & -1.92 & 0.00 \\
\hline CD59 & -1.01 & 0.03 & HIST1H2BK & -1.48 & 0.00 \\
\hline CD82 & -1.37 & 0.03 & $\mathrm{HIST} 2 \mathrm{H} 2 \mathrm{AA} 3$ & -1.13 & 0.03 \\
\hline CDKN2A & -3.20 & 0.00 & HIST2H4A & -2.11 & 0.00 \\
\hline CEACAM1 & -1.89 & 0.00 & HSPA1A & -1.83 & 0.02 \\
\hline CEACAMG & -1.71 & 0.05 & HSPB1 & -1.41 & 0.03 \\
\hline CGN & -1.06 & 0.02 & $\mathrm{HSPH} 1$ & -1.43 & 0.00 \\
\hline CHKA & -1.13 & 0.00 & IDH2 & -1.01 & 0.04 \\
\hline CHMP4B & -1.16 & 0.00 & IDS & -1.30 & 0.02 \\
\hline CLDN1 & -1.37 & 0.05 & $\mathrm{IF|}$ & -1.76 & 0.03 \\
\hline CLDN3 & -1.58 & 0.01 & IRF1 & -1.02 & 0.01 \\
\hline COL17A1 & -1.61 & 0.03 & KRT2O & -1.73 & 0.04 \\
\hline COTL1 & -1.61 & 0.00 & KRT23 & -4.08 & 0.00 \\
\hline CPNE1 & -1.05 & 0.00 & LAMC2 & -1.77 & 0.00 \\
\hline CPT1B & 1.13 & 0.00 & LFNG & -1.19 & 0.02 \\
\hline CTSA & -1.10 & 0.00 & LGALS1 & -2.88 & 0.00 \\
\hline CTSD & -1.02 & 0.03 & LINC01089 & -1.05 & 0.01 \\
\hline CTSH & -1.22 & 0.03 & LIPG & 1.38 & 0.00 \\
\hline CTSS & -2.46 & 0.00 & LPCAT2 & -1.11 & 0.01 \\
\hline CTSV & -1.81 & 0.00 & LRRC75A-AS1 & 1.01 & 0.04 \\
\hline CTSZ & -1.08 & 0.01 & LRRC8A & -1.23 & 0.00 \\
\hline CUEDC1 & -1.33 & 0.02 & LTBP3 & -1.37 & 0.01 \\
\hline CXXC5 & -1.24 & 0.01 & LY6E & -1.41 & 0.02 \\
\hline CYB5D1 & 1.24 & 0.00 & LY75 & -1.60 & 0.01 \\
\hline
\end{tabular}

Table 5 List of genes differentially expressed in the indicated cell lines (Continued) 
Table $\mathbf{5}$ List of genes differentially expressed in the indicated cell lines (Continued)

\begin{tabular}{|c|c|c|c|c|c|}
\hline gene_id & Log2 fold change & $p$ adj. & gene_id & Log2 fold change & $p$ adj. \\
\hline MACF1 & -1.23 & 0.01 & SPINK1 & -2.25 & 0.01 \\
\hline MALL & -1.20 & 0.03 & STAT1 & -1.06 & 0.01 \\
\hline MAP7D1 & -1.03 & 0.02 & SULT2B1 & -1.78 & 0.01 \\
\hline MAPRE3 & -1.11 & 0.02 & SUPT4H1 & 1.22 & 0.00 \\
\hline MDM2 & 1.37 & 0.00 & SYT7 & -1.82 & 0.00 \\
\hline MGLL & -1.35 & 0.01 & TCF7 & -1.03 & 0.04 \\
\hline MIR4435-2HG & -1.09 & 0.05 & TFF1 & 2.17 & 0.02 \\
\hline MMP14 & -1.53 & 0.02 & TGFBI & -2.43 & 0.00 \\
\hline MOCOS & 1.18 & 0.01 & TIMP2 & -2.16 & 0.01 \\
\hline MORC4 & 1.15 & 0.00 & TM4SF1 & -1.70 & 0.01 \\
\hline MUC20 & -1.64 & 0.02 & TMEM52 & 1.08 & 0.03 \\
\hline MYBL2 & -1.13 & 0.01 & TMPRSS4 & -1.35 & 0.04 \\
\hline MYL5 & 1.04 & 0.01 & TNFSF9 & 2.01 & 0.00 \\
\hline NABP1 & -1.35 & 0.01 & TRIM7 & 1.24 & 0.05 \\
\hline NDUFC2 & -1.22 & 0.00 & TSPAN6 & -1.49 & 0.00 \\
\hline OXR1 & -1.12 & 0.01 & TUBA4A & -1.11 & 0.00 \\
\hline PDP1 & -1.01 & 0.03 & TUBE1 & 1.12 & 0.01 \\
\hline PEA15 & -1.29 & 0.00 & TXNDC9 & -1.04 & 0.00 \\
\hline PFDN4 & -1.04 & 0.00 & UCA1 & -1.79 & 0.03 \\
\hline PIGT & -1.00 & 0.00 & UCP2 & -1.38 & 0.03 \\
\hline PLA2G6 & 1.17 & 0.00 & UNC13D & -1.81 & 0.01 \\
\hline PLS3 & -1.21 & 0.03 & VAMP8 & -1.09 & 0.00 \\
\hline PMEPA1 & -2.81 & 0.00 & VOPP1 & -1.13 & 0.01 \\
\hline PML & -1.04 & 0.00 & ZMYND8 & -1.11 & 0.00 \\
\hline POLE4 & -1.01 & 0.01 & ZNFX1 & -1.08 & 0.00 \\
\hline PPM1M & 1.46 & 0.00 & Hypermutated vs non-hypermutated & & \\
\hline PPP1R14D & -1.98 & 0.01 & ABHD12 & -1.10 & 0.00 \\
\hline PPP1R18 & -1.27 & 0.01 & АСОT8 & -1.09 & 0.00 \\
\hline PRADC1 & -1.19 & 0.01 & AHCYL2 & 1.00 & 0.01 \\
\hline PRAP1 & -1.79 & 0.04 & AKR1C3 & -1.52 & 0.02 \\
\hline QPCT & -1.89 & 0.02 & ALDH6A1 & 1.30 & 0.00 \\
\hline QPRT & -2.16 & 0.00 & AMN & 1.06 & 0.04 \\
\hline REG4 & 2.81 & 0.00 & ANXA6 & -1.94 & 0.00 \\
\hline ROMO1 & -1.14 & 0.01 & AOC1 & -1.96 & 0.00 \\
\hline RPL22L1 & 1.62 & 0.00 & ARHGEF10 & 1.15 & 0.04 \\
\hline RPL32P29 & 1.31 & 0.00 & ARL4C & -1.56 & 0.01 \\
\hline S100A11 & -1.62 & 0.00 & ATOX1 & -1.12 & 0.00 \\
\hline S100A2 & -2.35 & 0.00 & ATP5E & -1.31 & 0.00 \\
\hline S100A4 & -2.70 & 0.00 & ATP8B1 & 1.09 & 0.00 \\
\hline SDC4 & -1.30 & 0.00 & ATP9A & -1.77 & 0.00 \\
\hline SESN2 & 1.23 & 0.01 & B2M & -1.82 & 0.00 \\
\hline SGK2 & -1.40 & 0.04 & BCAS4 & -1.06 & 0.00 \\
\hline SLC2OA2 & -1.07 & 0.01 & BCL2L1 & -1.02 & 0.00 \\
\hline SLC2A1 & -1.22 & 0.04 & BNIP3 & 1.31 & 0.02 \\
\hline SLC39A5 & -1.93 & 0.02 & C15orf52 & -1.10 & 0.04 \\
\hline SLC6A6 & -1.40 & 0.00 & C20orf24 & -1.21 & 0.00 \\
\hline SNHG8 & 1.01 & 0.01 & C2orf54 & -2.00 & 0.00 \\
\hline SNORA73B & 1.12 & 0.02 & C7orf50 & -1.20 & 0.00 \\
\hline
\end{tabular}

Table 5 List of genes differentially expressed in the indicated cell lines (Continued) 
Table $\mathbf{5}$ List of genes differentially expressed in the indicated cell lines (Continued)

\begin{tabular}{|c|c|c|c|c|c|}
\hline gene_id & Log2 fold change & $p$ adj. & gene_id & Log2 fold change & $p$ adj. \\
\hline CAPG & -1.02 & 0.03 & GPC1 & -1.01 & 0.05 \\
\hline CCDC71L & -1.00 & 0.01 & GSN & -1.75 & 0.00 \\
\hline CD59 & -1.13 & 0.00 & HELZ2 & -1.02 & 0.01 \\
\hline CD82 & -1.31 & 0.01 & $\mathrm{HIST} 1 \mathrm{H} 2 \mathrm{BD}$ & -1.04 & 0.01 \\
\hline CDKN2A & -1.43 & 0.03 & $\mathrm{HIST} 2 \mathrm{H} 4 \mathrm{~A}$ & -1.38 & 0.00 \\
\hline CEACAM1 & -1.42 & 0.00 & HSD11B2 & -1.11 & 0.03 \\
\hline CFD & 1.65 & 0.00 & HSPB1 & -1.20 & 0.02 \\
\hline CHKA & -1.17 & 0.00 & IDS & -1.60 & 0.00 \\
\hline CHMP4B & -1.25 & 0.00 & IFI27 & -1.48 & 0.03 \\
\hline CLDN3 & -1.45 & 0.00 & $\mid \mathrm{FI} 27 \mathrm{~L} 2$ & -1.03 & 0.03 \\
\hline COL17A1 & -1.33 & 0.03 & $\mathrm{IF|6}$ & -1.86 & 0.00 \\
\hline COTL1 & -1.67 & 0.00 & IGFBP4 & -1.15 & 0.02 \\
\hline CPNE1 & -1.09 & 0.00 & IL33 & 1.58 & 0.04 \\
\hline CST3 & -1.29 & 0.00 & IRF1 & -1.28 & 0.00 \\
\hline CTSA & -1.01 & 0.00 & ISG15 & -1.13 & 0.04 \\
\hline CTSD & -1.27 & 0.00 & ITGA3 & -1.23 & 0.01 \\
\hline CTSH & -1.31 & 0.00 & ITGB5 & -1.09 & 0.00 \\
\hline CTSS & -2.26 & 0.00 & $\mathrm{KIFC3}$ & -1.20 & 0.04 \\
\hline CTSV & -2.11 & 0.00 & KLK6 & -2.00 & 0.00 \\
\hline CTSZ & -1.05 & 0.00 & KRT2O & -1.56 & 0.02 \\
\hline CUEDC1 & -1.59 & 0.00 & KRT23 & -3.88 & 0.00 \\
\hline CXXC5 & -1.01 & 0.01 & KRT80 & -1.58 & 0.00 \\
\hline CYB5D1 & 1.17 & 0.00 & LAMC2 & -1.57 & 0.00 \\
\hline CYTOR & -1.42 & 0.00 & LFNG & -1.23 & 0.00 \\
\hline DBNDD2 & -1.06 & 0.00 & LGALS1 & -2.44 & 0.00 \\
\hline DCBLD2 & -2.20 & 0.00 & LIPG & 1.38 & 0.00 \\
\hline DGAT2 & -1.04 & 0.01 & LITAF & -1.20 & 0.01 \\
\hline DHRS3 & -1.09 & 0.01 & LTBP3 & -1.63 & 0.00 \\
\hline DNTTIP1 & -1.23 & 0.00 & LTBP4 & 1.17 & 0.00 \\
\hline DYNLRB1 & -1.25 & 0.00 & LY6E & -1.74 & 0.00 \\
\hline EDN1 & -1.27 & 0.03 & LY6G6D & -2.03 & 0.01 \\
\hline EHD1 & -1.01 & 0.00 & LY75 & -1.60 & 0.00 \\
\hline EMP1 & -1.60 & 0.00 & MAPRE3 & -1.23 & 0.00 \\
\hline EPB41L1 & -1.06 & 0.00 & MBOAT2 & -1.03 & 0.02 \\
\hline EPS8L1 & -1.20 & 0.00 & MCRIP1 & -1.02 & 0.00 \\
\hline FAH & -1.28 & 0.00 & MDK & -1.36 & 0.01 \\
\hline FAM84B & -1.10 & 0.02 & MDM2 & 1.09 & 0.00 \\
\hline FCGRT & -1.60 & 0.00 & MELTF & -1.18 & 0.01 \\
\hline $\mathrm{FECH}$ & 1.27 & 0.00 & MGLL & -1.20 & 0.00 \\
\hline FKBP10 & -1.41 & 0.04 & MIR4435-2HG & -1.47 & 0.00 \\
\hline FKBP1A & -1.16 & 0.00 & MMP14 & -1.64 & 0.00 \\
\hline FUNDC2 & -1.04 & 0.00 & MOCOS & 1.13 & 0.00 \\
\hline FUNDC2P1 & -1.08 & 0.00 & MOSPD1 & -1.01 & 0.00 \\
\hline FZD7 & -1.19 & 0.00 & MUC20 & -1.58 & 0.00 \\
\hline GABARAPL1 & -1.35 & 0.01 & MYBL2 & -1.06 & 0.00 \\
\hline GABRE & -1.53 & 0.00 & NABP1 & -1.05 & 0.01 \\
\hline GCAT & 1.01 & 0.01 & NDUFC2 & -1.11 & 0.00 \\
\hline GNE & -1.03 & 0.02 & PDP1 & -1.16 & 0.00 \\
\hline
\end{tabular}

Table 5 List of genes differentially expressed in the indicated cell lines (Continued) 
Table $\mathbf{5}$ List of genes differentially expressed in the indicated cell lines (Continued)

\begin{tabular}{|c|c|c|c|c|c|}
\hline gene_id & Log2 fold change & $p$ adj. & gene_id & Log2 fold change & $p$ adj \\
\hline PEA15 & -1.30 & 0.00 & TMPRSS4 & -1.35 & 0.01 \\
\hline PFDN4 & -1.20 & 0.00 & TNFRSF14-AS1 & 1.19 & 0.02 \\
\hline PHLDB1 & -1.05 & 0.05 & TNFRSF1B & -1.44 & 0.00 \\
\hline PLAUR & -1.18 & 0.00 & TRIB1 & -1.11 & 0.02 \\
\hline PLTP & -1.53 & 0.03 & TRIM7 & 1.08 & 0.04 \\
\hline PMEPA1 & -2.92 & 0.00 & TSC22D1 & -1.01 & 0.01 \\
\hline PML & -1.03 & 0.00 & TUBA4A & -1.01 & 0.00 \\
\hline POLD4 & -1.06 & 0.00 & TUBB2A & -1.49 & 0.00 \\
\hline PPP1R14D & -2.05 & 0.00 & TUBE1 & 1.12 & 0.00 \\
\hline PRDX5 & -1.09 & 0.00 & UCA1 & -1.49 & 0.03 \\
\hline PRR15 & -1.06 & 0.03 & UCP2 & -1.16 & 0.02 \\
\hline PRSS23 & -1.09 & 0.02 & VAMP8 & -1.10 & 0.00 \\
\hline PSMA7 & -1.00 & 0.00 & VEGFB & -1.25 & 0.00 \\
\hline PSMB10 & -1.27 & 0.00 & VOPP1 & -1.27 & 0.00 \\
\hline $\mathrm{QPCT}$ & -2.23 & 0.00 & VSIR & -1.68 & 0.00 \\
\hline QPRT & -1.32 & 0.05 & ZFP90 & 1.13 & 0.00 \\
\hline RASL11A & -1.19 & 0.04 & ZMYND8 & -1.07 & 0.00 \\
\hline REG4 & 2.99 & 0.00 & EVOLVING-CRC vs STABLE-CRC & & \\
\hline RGCC & -2.97 & 0.00 & ABCB6 & -1.00 & 0.04 \\
\hline RGMB & 1.49 & 0.00 & $\mathrm{AHI}$ & 1.04 & 0.02 \\
\hline $\mathrm{RHOD}$ & -1.22 & 0.01 & ANXA6 & -1.83 & 0.02 \\
\hline ROMO1 & -1.28 & 0.00 & ATOX1 & -1.07 & 0.01 \\
\hline RPL22L1 & 1.12 & 0.01 & B2M & -1.57 & 0.00 \\
\hline RTFDC1 & -1.04 & 0.00 & CPNE1 & -1.12 & 0.00 \\
\hline S100A11 & -1.65 & 0.00 & FAT1 & 1.09 & 0.04 \\
\hline S100A2 & -2.87 & 0.00 & GTF3A & -1.09 & 0.03 \\
\hline S100A4 & -1.55 & 0.02 & IGFBP6 & -2.01 & 0.02 \\
\hline SDC4 & -1.30 & 0.00 & IRF1 & -1.05 & 0.02 \\
\hline SLC20A2 & -1.01 & 0.00 & LGALS1 & -2.45 & 0.00 \\
\hline SLC2A1 & -1.42 & 0.00 & LY6E & -1.47 & 0.02 \\
\hline SLC2A4RG & -1.04 & 0.00 & PAM & -1.27 & 0.03 \\
\hline SLC2A8 & -1.04 & 0.00 & PEA15 & -1.12 & 0.01 \\
\hline SLC39A5 & -1.70 & 0.01 & PLA2G2A & 2.56 & 0.01 \\
\hline SLCO1B3 & 1.46 & 0.05 & PSMB10 & -1.05 & 0.02 \\
\hline SMAD3 & -1.08 & 0.00 & S100A2 & -1.96 & 0.00 \\
\hline SMIM22 & -1.19 & 0.01 & SDC4 & -1.04 & 0.05 \\
\hline SNORA73B & 1.00 & 0.01 & SLC7A11 & 1.63 & 0.01 \\
\hline SPINK1 & -2.72 & 0.00 & TGFBI & -1.50 & 0.04 \\
\hline SULT2B1 & -1.60 & 0.00 & TIMP2 & -1.82 & 0.03 \\
\hline SYT7 & -1.55 & 0.00 & TUBA1A & -1.71 & 0.05 \\
\hline TACSTD2 & -2.32 & 0.00 & TUBB2A & -1.55 & 0.03 \\
\hline TCF7 & -1.18 & 0.00 & TUBE1 & 1.27 & 0.01 \\
\hline TGFBI & -2.28 & 0.00 & VEGFA & 1.08 & 0.05 \\
\hline TIMP2 & -2.59 & 0.00 & & & \\
\hline TM4SF1 & -1.78 & 0.00 & & & \\
\hline TMEM176A & -2.16 & 0.00 & & & \\
\hline TMEM176B & -1.85 & 0.01 & & & \\
\hline TMEM185A & -1.07 & 0.00 & & & \\
\hline
\end{tabular}

Table 5 List of genes differentially expressed in the indicated cell lines (Continued) 
from adaption previously experienced in the patient as a mechanism of escape from negative pressure of the immune system related to the elevated neoantigens' production rate.

\section{Conclusions}

In summary, we identified and functionally highlighted CRC subsets characterized by slow and fast genome evolvability. CRCs carrying alterations in genes involved in DNA repair (including $M L H 1, M S H 2, M S H 6, M U T Y H$, EXO1, and POLE) display dynamic neoantigen patterns that fluctuate over time. Furthermore, we find that in CRC cells and patient-derived tumor xenografts, DNA repair defects leading to high mutational burden and neoantigen evolvability are associated with inactivation or downregulation of antigen-presentation functions. Longitudinal monitoring of the neoantigen landscape of CRC and other tumor types may have clinical implications. While tracking time-dependent neoantigen evolution in the tissue of cancer patients might be difficult or impossible to achieve, monitoring predicted neoantigens in circulating tumor DNA is already within reach. Accordingly, longitudinal liquid biopsies could be deployed to assess whether and how time and/or therapeutic regimens affect the mutational burden and the neoantigen profiles in individual patients. Neoantigen clonality profiles could be valuable to develop specific vaccines and deploy immunomodulatory molecules in the context of precision oncology.

\section{Additional files}

Additional file 1: Supplementary figures S1-S15. (PDF 2606 kb)

Additional file 2: Single nucleotide variants and frameshifts of 64 CRC cell lines at TO. (XLSX $9992 \mathrm{~kb}$ )

\section{Acknowledgements}

We acknowledge Francesco Galimi for providing gDNA and RNA from PDXs. We thank Daniela Cantarella for performing RNAseq. We thank all members of our laboratory for valuable suggestions and critical review of the data and the manuscript, as well as all laboratory technicians for their help.

\section{Authors' contributions}

$A B, G R, A L$, and NA conceived and designed the study. AL, NA, AM, CC, VA, $M M, A B a r t$, and GG performed the experiments. GR, GC, CN, LN, and Cl performed the bioinformatics analysis. $A B, G R, A L$, and $G G$ interpreted the data. LT and ABer provided samples and interpreted the data. $A B, G R$, and $\mathrm{AL}$ wrote the manuscript with input from all authors. All authors read and approved the final manuscript.

\section{Funding}

The research leading to these results has received funding from AIRC IG program: FONDAZIONE AIRC IG 2018 - ID. 21923 project -

PI: AB; FONDAZIONE AIRC IG 2018 - ID. 21407 project - PI: FDN; ; FONDAZIONE AIRC IG 2017 - ID. 20697 project - PI: ABer; FONDAZIONE AIRC IG 2016 - ID. 18532 project - PI: LT. The research leading to these results has also received funding from FONDAZIONE AIRC under 5 per Mille 2018 ID. 21091 program - PI: AB; GL: FDN, EM, LT, ABer. Fondazione Piemontese per la Ricerca sul Cancro-ONLUS, 5× 1000 Ministero della Salute 2011, 2014 and 2015 (AB, LT, FDN, EM). RC 2017 Ministero della Salute (AB, LT, FDN and
EM). Partly funded also by Fondo per la Ricerca Locale (ex 60\%), Università di Torino, 2017 (AL and FDN). This work was also supported by the European Community's H2020 grant agreement no. 635342-2 MoTriColor (AB), the European Community's Seventh Framework Programme under grant agreement n. 602901 MErCurRIC (AB), the European Research Council Consolidator Grant 724748 - BEAT (ABer) and TRANSCAN-2 JTC 2014 Tactic (LT).

\section{Availability of data and materials}

Detailed mutational characterization of the 64 CRC cell lines is available in Additional file 2. NGS data from cell lines used in the current study are available in the European Nucleotide Archive (ENA) with the following accession code PRJEB33045.

\section{Ethics approval and consent to participate}

All animal procedures were approved by the Ethical Commission of Candiolo Cancer Institute IRCCS, of the University of Turin and by the Italian Ministry of Health. They were performed in accordance with institutional guidelines and international law and policies. The number of mice included in the experiments, the inclusion/exclusion criteria, and the observed tumor size limits were based on institutional guidelines. Guidelines limited us to using 6-8-week-old female and male NOD-SCID mice. Mice were obtained from Charles River Tumor samples, and matched normal samples were obtained from patients treated by liver metastasectomy at the Candiolo Cancer Institute (Candiolo, Torino, Italy), Mauriziano Umberto I Hospital (Torino) and San Giovanni Battista Hospital (Torino). All patients provided informed consent, samples were procured, and the study was conducted under the approval of the Review Boards of the Institutions (Ethics protocol No. 001IRCC-00IIS-10, project "PROFILING").

\section{Consent for publication}

Not applicable.

\section{Competing interests}

G. Germano has an ownership interest in Neophore. A. Bardelli has ownership interest in Phoremost and Neophore and is a consultant/advisory board member for Phoremost and Neophore. The remaining authors declare that they have no competing interests.

\section{Author details}

${ }^{1}$ Candiolo Cancer Institute, FPO-IRCCS, 10060 Candiolo (TO), Italy.

²Department of Oncology, University of Turin, 10060 Candiolo (TO), Italy.

${ }^{3}$ FIRC Institute of Molecular Oncology (IFOM), 20139 Milan, Italy.

Received: 17 January 2019 Accepted: 13 June 2019

Published online: 28 June 2019

\section{References}

1. Overman MJ, McDermott R, Leach JL, Lonardi S, Lenz HJ, Morse MA, et al. Nivolumab in patients with metastatic DNA mismatch repair-deficient or microsatellite instability-high colorectal cancer (CheckMate 142): an openlabel, multicentre, phase 2 study. Lancet Oncol. 2017;18(9):1182-91.

2. Gibney GT, Weiner LM, Atkins MB. Predictive biomarkers for checkpoint inhibitor-based immunotherapy. Lancet Oncol. 2016;17(12):e542-e51.

3. Ribas A, Wolchok JD. Cancer immunotherapy using checkpoint blockade. Science. 2018;359(6382):1350-5

4. Cristescu R, Mogg R, Ayers M, Albright A, Murphy E, Yearley J, et al. Pantumor genomic biomarkers for PD-1 checkpoint blockade-based immunotherapy. Science. 2018;362(6411):1-10.

5. Lee $\mathrm{CH}$, Yelensky $\mathrm{R}$, Jooss K, Chan TA. Update on tumor neoantigens and their utility: why it is good to be different. Trends Immunol. 2018; 39(7):536-48

6. Samstein RM, Lee $\mathrm{CH}$, Shoushtari AN, Hellmann MD, Shen $\mathrm{R}$, Janjigian $Y$, et al. Tumor mutational load predicts survival after immunotherapy across multiple cancer types. Nat Genet. 2019;51(2):202-6.

7. Shlien A, Campbell BB, de Borja R, Alexandrov LB, Merico D, Wedge D, et al. Combined hereditary and somatic mutations of replication error repair genes result in rapid onset of ultra-hypermutated cancers. Nat Genet. 2015;47(3):257-62.

8. Turajlic S, Litchfield K, Xu H, Rosenthal R, McGranahan N, Reading JL, et al. Insertion-and-deletion-derived tumour-specific neoantigens and the 
immunogenic phenotype: a pan-cancer analysis. Lancet Oncol. 2017; 18(8):1009-21.

9. Germano G, Lamba S, Rospo G, Barault L, Magrì A, Maione F, et al. Inactivation of DNA repair triggers neoantigen generation and impairs tumour growth. Nature. 2017;552(7683):116-20.

10. Conway T, Wazny J, Bromage A, Tymms M, Sooraj D, Williams ED, et al. Xenome--a tool for classifying reads from xenograft samples. Bioinformatics. 2012;28(12):i172-8

11. Li H, Durbin R. Fast and accurate long-read alignment with BurrowsWheeler transform. Bioinformatics. 2010;26(5):589-95.

12. Li H, Handsaker B, Wysoker A, Fennell T, Ruan J, Homer N, et al. The sequence alignment/map format and SAMtools. Bioinformatics. 2009; 25(16):2078-9

13. Siravegna G, Mussolin B, Buscarino M, Corti G, Cassingena A, Crisafulli G, et al. Clonal evolution and resistance to EGFR blockade in the blood of colorectal cancer patients. Nat Med. 2015;21(7):795-801.

14. Germano G, Amirouchene-Angelozzi N, Rospo G, Bardelli A. The clinical impact of the genomic landscape of mismatch repair-deficient cancers. Cancer Discov. 2018;8(12):1518-28.

15. Szolek A, Schubert B, Mohr C, Sturm M, Feldhahn M, Kohlbacher O. OptiType: precision HLA typing from next-generation sequencing data. Bioinformatics. 2014;30(23):3310-6.

16. Andreatta M, Nielsen M. Gapped sequence alignment using artificial neural networks: application to the MHC class I system. Bioinformatics. 2016;32(4):511-7.

17. Díaz-Gay M, Vila-Casadesús M, Franch-Expósito S, Hernández--llán E, Lozano JJ, Castellví-Bel S. Mutational Signatures in Cancer (MuSiCa): a web application to implement mutational signatures analysis in cancer samples. BMC Bioinformatics. 2018;19(1):224.

18. Alexandrov LB, Nik-Zainal S, Wedge DC, Aparicio SA, Behjati S, Biankin AV, et al. Signatures of mutational processes in human cancer. Nature. 2013; 500(7463):415-21.

19. Hirsch HR, Engelberg J. Determination of the cell doubling-time distribution from culture growth-rate data. J Theor Biol. 1965;9(2):297-302.

20. Wang K, Singh D, Zeng Z, Coleman SJ, Huang Y, Savich GL, et al. MapSplice: accurate mapping of RNA-seq reads for splice junction discovery. Nucleic Acids Res. 2010;38(18):e178.

21. Li B, Dewey CN. RSEM: accurate transcript quantification from RNA-Seq data with or without a reference genome. BMC Bioinformatics. 2011;12:323.

22. Love Ml, Huber W, Anders S. Moderated estimation of fold change and dispersion for RNA-seq data with DESeq2. Genome Biol. 2014;15(12):550.

23. Reimand J, Arak T, Adler P, Kolberg L, Reisberg S, Peterson H, et al. g: Profiler-a web server for functional interpretation of gene lists (2016 update). Nucleic Acids Res. 2016;4((W1):W83-9.

24. Galimi F, Torti D, Sassi F, Isella C, Corà D, Gastaldi S, et al. Genetic and expression analysis of MET, MACC1, and HGF in metastatic colorectal cancer: response to met inhibition in patient xenografts and pathologic correlations. Clin Cancer Res. 2011;17(10):3146-56.

25. Siravegna G, Lazzari L, Crisafulli G, Sartore-Bianchi A, Mussolin B, Cassingena $A$, et al. Radiologic and genomic evolution of individual metastases during HER2 blockade in colorectal cancer. Cancer Cell. 2018;34(1):148-62 e7.

26. Corti G, Bartolini A, Crisafulli G, Novara L, Rospo G, Montone M, et al. A Genomic Analysis Workflow for Colorectal Cancer Precision Oncology. Clin Colorectal Cancer. 2019;18(2):91-101.e3.

27. Network CGA. Comprehensive molecular characterization of human colon and rectal cancer. Nature. 2012;487(7407):330-7.

28. Goellner EM, Putnam CD, Kolodner RD. Exonuclease 1-dependent and independent mismatch repair. DNA Repair (Amst). 2015;32:24-32.

29. Boiteux S, Coste F, Castaing B. Repair of 8-oxo-7,8-dihydroguanine in prokaryotic and eukaryotic cells: properties and biological roles of the Fpg and OGG1 DNA N-glycosylases. Free Radic Biol Med. 2017;107:179-201.

30. Dallosso AR, Dolwani S, Jones N, Jones S, Colley J, Maynard J, et al. Inherited predisposition to colorectal adenomas caused by multiple rare alleles of MUTYH but not OGG1, NUDT1, NTH1 or NEIL 1, 2 or 3. Gut. 2008;57(9):1252-5.

31. Sim NL, Kumar P, Hu J, Henikoff S, Schneider G, Ng PC. SIFT web server: predicting effects of amino acid substitutions on proteins. Nucleic Acids Res. 2012;40(Web Server issue:W452-7.

32. Adzhubei IA, Schmidt S, Peshkin L, Ramensky VE, Gerasimova A, Bork P, et al. A method and server for predicting damaging missense mutations. Nat Methods. 2010;7(4):248-9.
33. Viel A, Bruselles A, Meccia E, Fornasarig M, Quaia M, Canzonieri V, et al. A specific mutational signature associated with DNA 8-oxoguanine persistence in MUTYH-defective colorectal cancer. EBioMedicine. 2017; 20:39-49.

34. Gajewski TF, Schreiber H, Fu YX. Innate and adaptive immune cells in the tumor microenvironment. Nat Immunol. 2013;14(10):1014-22.

35. Bertotti A, Papp E, Jones S, Adleff V, Anagnostou V, Lupo B, et al. The genomic landscape of response to EGFR blockade in colorectal cancer. Nature. 2015;526(7572):263-7.

36. Guinney J, Dienstmann R, Wang X, de Reyniès A, Schlicker A, Soneson C, et al. The consensus molecular subtypes of colorectal cancer. Nat Med. 2015; 21(11):1350-6

37. Isella C, Terrasi A, Bellomo SE, Petti C, Galatola G, Muratore A, et al. Stromal contribution to the colorectal cancer transcriptome. Nat Genet. 2015:47(4):312-9.

38. Sinicrope FA, Rego RL, Halling KC, Foster N, Sargent DJ, La Plant B, et al. Prognostic impact of microsatellite instability and DNA ploidy in human colon carcinoma patients. Gastroenterology. 2006;131(3):729-37.

39. Tian S, Roepman P, Popovici V, Michaut M, Majewski I, Salazar R, et al. A robust genomic signature for the detection of colorectal cancer patients with microsatellite instability phenotype and high mutation frequency. J Pathol. 2012;228(4):586-95.

40. Dagogo-Jack I, Shaw AT. Tumour heterogeneity and resistance to cancer therapies. Nat Rev Clin Oncol. 2018;15(2):81-94.

41. Govindan R, Ding L, Griffith M, Subramanian J, Dees ND, Kanchi KL, et al. Genomic landscape of non-small cell lung cancer in smokers and neversmokers. Cell. 2012;150(6):1121-34.

42. Alexandrov LB, Ju YS, Haase K, Van Loo P, Martincorena I, Nik-Zainal S, et al. Mutational signatures associated with tobacco smoking in human cancer. Science. 2016;354(6312):618-22.

43. Hodis E, Watson IR, Kryukov GV, Arold ST, Imielinski M, Theurillat JP, et al. A landscape of driver mutations in melanoma. Cell. 2012;150(2):251-63.

44. Network CGAR. Comprehensive molecular profiling of lung adenocarcinoma. Nature. 2014;511(7511):543-50.

45. Network CGA. Genomic classification of cutaneous melanoma. Cell. 2015;161(7):1681-96.

46. Yang W, Soares J, Greninger P, Edelman EJ, Lightfoot $\mathrm{H}$, Forbes $\mathrm{S}$, et al. Genomics of drug sensitivity in cancer (GDSC): a resource for therapeutic biomarker discovery in cancer cells. Nucleic Acids Res. 2013;41(Database issue):D955-61.

47. Garnett MJ, Edelman EJ, Heidorn SJ, Greenman CD, Dastur A, Lau KW, et al. Systematic identification of genomic markers of drug sensitivity in cancer cells. Nature. 2012;483(7391):570-5.

48. Barretina J, Caponigro G, Stransky N, Venkatesan K, Margolin AA, Kim S, et al. The Cancer Cell Line Encyclopedia enables predictive modelling of anticancer drug sensitivity. Nature. 2012;483(7391):603-7.

49. Iorio F, Knijnenburg TA, Vis DJ, Bignell GR, Menden MP, Schubert M, et al. A landscape of pharmacogenomic interactions in cancer. Cell. 2016; 166(3):740-54.

50. Vanden Heuvel JP, Bullenkamp J, Biology RPC. Registered report: systematic identification of genomic markers of drug sensitivity in cancer cells. Elife. 2016;5:1-19.

51. Liu Y, Mi Y, Mueller T, Kreibich S, Williams EG, Van Drogen A, et al. Multiomic measurements of heterogeneity in HeLa cells across laboratories. Nat Biotechnol. 2019:37(3):314-22.

52. Ben-David U, Siranosian B, Ha G, Tang H, Oren Y, Hinohara K, et al. Genetic and transcriptional evolution alters cancer cell line drug response. Nature. 2018:560(7718):325-30

53. Ben-David U, Ha G, Tseng YY, Greenwald NF, Oh C, Shih J, et al. Patientderived xenografts undergo mouse-specific tumor evolution. Nat Genet. 2017:49(11):1567-75

\section{Publisher's Note}

Springer Nature remains neutral with regard to jurisdictional claims in published maps and institutional affiliations. 